\title{
Chapitre 7 \\ La sûreté pour les réacteurs de recherche français
}

\subsection{Principes, concepts, démarches et objectifs généraux de sûreté}

Le présent chapitre vise à exposer les principes, concepts, démarches et objectifs généraux de sûreté qui ont guidé la conception et l'exploitation des réacteurs de recherche français. Il vise aussi à souligner leurs évolutions au fil du temps qui, de façon générale, ont conduit à un rapprochement avec les pratiques adoptées pour les réacteurs électronucléaires, y compris ceux relatifs au réacteur EPR. Quelques spécificités des réacteurs de recherche seront mises en avant, parmi lesquelles la plus notable est la prise en compte, dès la conception de certains réacteurs de recherche français dans les années 1960, d'accidents impliquant une fusion de combustible.

Les dispositions retenues en matière de sûreté nucléaire et de radioprotection pour la conception et l'exploitation des réacteurs nucléaires, qu'ils soient électrogènes ou de recherche, doivent tendre à minimiser le nombre d'incidents, limiter les possibilités d'apparition d'accidents et satisfaire un objectif fondamental en matière de sûreté, tel qu'énoncé notamment dans le document SF-1 de l'AIEA, à savoir « protéger les personnes et l'environnement contre les effets nocifs des rayonnements ionisants ». Cet objectif est bien évidemment inscrit dans la règlementation française, plus précisément dans le code de la santé publique (article L. 1333-1) et dans le code de l'environnement (L. 110-1).

Les dispositions de conception visent de multiples aspects : les caractéristiques intrinsèques du réacteur (par exemple en matière de neutronique du cœur), l'architecture 
générale des systèmes, les redondances et diversifications matérielles, les protections contre les agressions internes et externes, les protections radiologiques, le choix des fluides mis en œuvre et les dispositions de gestion et de traitement des effluents, le choix des matériaux, etc. L'adoption de pratiques éprouvées pour la conception et la fabrication des équipements permet de bénéficier d'un retour d'expérience favorable. L'adoption des meilleures techniques disponibles ${ }^{166}$ est aussi souhaitable, dans la mesure où elles sont applicables à l'installation concernée.

Le document SF-1 et, en France, les textes précités ainsi que la loi TSN et l'arrêté "INB », énoncent également un certain nombre de grands principes, allant de la responsabilité première de l'exploitant à la prévention des accidents et à la limitation de leurs conséquences s'ils devaient néanmoins survenir, introduisant le principe de défense en profondeur.

L'objectif fondamental rappelé ci-dessus est généralement décliné en objectifs généraux de sûreté exprimés de façon qualitative à l'égard des différents événements étudiés pour une installation, selon leurs fréquences estimées d'occurrence. Pour le projet du réacteur Jules Horowitz, ils ont été formulés de manière globalement similaire à celle adoptée par exemple pour l'EPR. Ainsi, pour les incidents les plus fréquents envisageables, il doit être fait en sorte que leurs conséquences non seulement ne nécessitent aucune contre-mesure pour les personnes du public et pour l'environnement mais restent aussi dans l'enveloppe des autorisations de rejets gazeux et liquides. Pour les accidents les plus graves étudiés, avec fusion du cœur, il doit être fait en sorte que leurs conséquences ne nécessitent qu'un recours à des contre-mesures très limitées, pour les personnes du public et pour l'environnement, en termes d'étendue et de durée (pas de relogement permanent, pas d'évacuation d'urgence au-delà du voisinage immédiat de l'installation, mise à l'abri limitée...). En d'autres termes ${ }^{167}$, il convient d'éviter :

- les rejets radioactifs précoces qui imposeraient des mesures d'urgence hors du site mais sans qu'il y ait assez de temps pour les mettre en œuvre ;

- les rejets radioactifs de grande ampleur qui imposeraient des mesures de protection qui ne pourraient pas être (suffisamment) limitées dans l'espace ou dans le temps.

Certains aspects de ces objectifs généraux de sûreté sont développés au paragraphe 7.1.4.

Les réacteurs de recherche, comme les réacteurs électronucléaires, présentent des risques associés aux matières radioactives qu'ils mettent en œuvre. Des rayonnements ionisants sont émis lors des réactions de fission ainsi que par les produits radioactifs formés lors de ces réactions de fission, ou par activation. La première voie possible d'exposition est l'irradiation directe par la source radioactive que constitue le réacteur nucléaire ou les circuits associés. Pour s'en protéger, des dispositions sont mises en œuvre

166. Cette notion est précisée dans l'annexe I de l'arrêté du 26 avril 2011 relatif à la mise en œuvre des meilleures techniques disponibles prévue par l'article R. 512-8 du code de l'environnement.

167. Ces objectifs seront formalisés dans la Directive 2014/87/EURATOM du Conseil du 8 juillet 2014 modifiant la directive 2007/71/Euratom établissant un cadre communautaire pour la sûreté nucléaire des installations nucléaires. 
(éloignement de la source, mise en place de matériaux absorbants tels que le plomb, le béton ou l'eau). Une autre voie possible d'exposition est la contamination résultant d'une dispersion de substances radioactives dans l'atmosphère. Les dispositions prises pour assurer le confinement de ces substances dans les conditions normales ou accidentelles de fonctionnement sont à cet égard fondamentales.

La sûreté d'un réacteur de recherche repose sur différents principes, concepts et démarches, qui ne sont pas en eux-mêmes spécifiques d'un tel réacteur :

- l'interposition de plusieurs «barrières » physiques de confinement entre les substances radioactives d'une part, les travailleurs, les personnes du public et l'environnement d'autre part, visant à assurer le confinement de ces substances. Ainsi, à l'égard des substances radioactives du cœur du réacteur, les « barrières » sont généralement au nombre de trois (des spécificités seront signalées plus loin pour des réacteurs de recherche) :

- les gaines des éléments combustibles,

- l'enveloppe du circuit de refroidissement du cœur ou de la piscine du réacteur,

- le bâtiment abritant le cœur du réacteur,

- la mise en place de dispositions permettant d'assurer les trois fonctions fondamentales de sûreté ${ }^{168}$, que sont :

- la maîtrise des réactions nucléaires en chaîne,

- l'évacuation de la puissance thermique issue des substances radioactives et des réactions nucléaires,

- le confinement des substances radioactives,

- l'adoption, pour les systèmes les plus importants pour la sûreté (visant notamment à assurer les deux premières fonctions fondamentales de sûreté), d'un principe de redondance, voire de diversification technologique, de façon à obtenir une fiabilité adéquate pour ces systèmes. Cela est notamment le cas pour les systèmes de protection et de sauvegarde d'un réacteur (qui interviennent dans la défense en profondeur) ;

- l'adoption d'une démarche de sûreté déterministe ${ }^{169}$ fondée sur une analyse, intégrant des conservatismes appropriés, d'un certain nombre d'événements ${ }^{170}$ postulés (liés à des défaillances propres de l'installation [erreurs humaines comprises],

168. Les deux premières de ces trois fonctions de sûreté contribuant à la tenue des « barrières » de confinement.

169. Démarche qui, au fil de ses évolutions, a pris en compte de façon indirecte des considérations probabilistes, notamment par le classement des conditions de fonctionnement en catégories selon la fréquence (ou probabilité) estimée des initiateurs ; les règles d'études en dépendent (cumul ou non d'une défaillance unique, façon de prendre en compte les incertitudes, critères à respecter pour les équipements...).

170. Événements initiateurs postulés dans les documents de l'AIEA, événements déclencheurs dans la règlementation française. 
à des agressions internes ou externes - voir le paragraphe 7.1.2), même si des études probabilistes peuvent apporter des éclairages utiles (voir plus loin). La déclinaison aux réacteurs de recherche de la démarche déterministe nécessite toutefois des analyses au cas par cas (notamment pour ce qui concerne l'établissement de la liste des événements postulés) compte tenu des risques très variés que présentent ces réacteurs.

Il est donc recherché que l'étanchéité des gaines des éléments combustibles (plaques, crayons), très souvent réalisés en alliage d'aluminium, soit assurée au moins dans les situations correspondant aux événements postulés dont la fréquence estimée est la plus élevée. Cela suppose que les transferts de chaleur entre le combustible et le caloporteur soient maîtrisés (ou plus précisément le rapport entre la puissance dégagée par le combustible et le débit du fluide de refroidissement), faute de quoi les températures des gaines s'accroîtraient irrémédiablement jusqu'à entraîner leur déformation, leur rupture, voire leur fusion (l'aluminium fond à $660^{\circ} \mathrm{C}$ ).

Par ailleurs, le cœur d'un réacteur est susceptible d'être le siège d'augmentations importantes ou de pics de puissance. Si la réaction en chaîne n'est pas maîtrisée, le dégagement d'énergie peut alors conduire à la fusion du combustible.

Il est rappelé que, dans un réacteur nucléaire, la maîtrise de la réactivité repose sur deux types d'éléments :

- les caractéristiques neutroniques intrinsèques au cœur, liées notamment à la nature du combustible et à celle du fluide réfrigérant, ainsi qu'à la géométrie du cœur (qui détermine notamment les fuites neutroniques) : proportion de neutrons différés (notée $\beta$ et exprimée en pcm), contre-réactions liées à l'effet Doppler dans le combustible et à l'effet de dilatation ou de contraction des structures et du fluide réfrigérant... À titre d'illustration, quelques valeurs de ces caractéristiques sont données, au paragraphe 7.2 , pour différents types de réacteurs de recherche et de réacteurs de puissance ;

- des éléments ajoutés, (barres, plaques) à base de matériaux absorbant les neutrons qui peuvent être insérés ou retirés du cœur manuellement, ou automatiquement en cas de dépassement de seuils associés à certains paramètres de fonctionnement du réacteur.

Les choix et les études de conception doivent viser à ce que ces éléments permettent d'assurer le meilleur comportement possible du réacteur en cas de perturbations telles que, par exemple, des insertions de réactivité. L'objectif est d'éviter autant que possible une prompte-criticité ${ }^{171}$, et d'assurer dans les situations d'arrêt, avec les absorbants insérés dans le cœur (hormis quelques absorbants de sécurité que l'on maintient en position extraite ${ }^{172}$ ), une marge suffisante (réactivité négative, ou antiréactivité) par rapport à l'atteinte d'un état critique. De plus, il doit être fait en sorte (par le nombre

171. La prompte-criticité est atteinte si la réactivité du cœur, contre-réactions prises en compte, devient supérieure à la proportion de neutrons différés.

172. Par exemple pour pouvoir apporter de l'antiréactivité en cas d'erreur lors d'un rechargement de cœur. 
d'absorbants et le " poids » en réactivité de chacune d'entre elles) que, lorsqu'un arrêt du réacteur est provoqué, cet arrêt puisse être assuré même en cas de non-chute de l'absorbant le plus efficace.

\subsubsection{Le principe de défense en profondeur appliqué aux réacteurs de recherche}

Le principe de défense en profondeur a été présenté dans ses grandes lignes au chapitre 3.

Certaines particularités de la défense en profondeur pour les réacteurs de recherche méritent toutefois d'être développées ici :

- le premier niveau de la défense en profondeur, qui vise à prévenir les anomalies de fonctionnement et les défaillances des structures, systèmes et composants (SSC), suppose notamment la qualité de la conception, de la fabrication de ces SSC et de l'exploitation du réacteur ( $y$ compris la maintenance préventive). Le maintien de cette qualité peut nécessiter une attention particulière pour les réacteurs de recherche $^{173}$, pour plusieurs raisons :

- leur durée d'exploitation peut être importante et faire l'objet de demandes d'extension au-delà de ce qui avait été prévu lors de la conception,

- de ce fait, les risques associés au vieillissement des matériels et à leur obsolescence peuvent être à redouter,

- le nécessaire renouvellement du personnel d'exploitation comporte des risques de défaillances dans la transmission des connaissances, cela pouvant conduire à l'émergence d'erreurs d'exploitation, voire d'événements parfois significatifs ${ }^{174}$,

- les réacteurs de recherche peuvent connaître des phases d'inutilisation avec éventuellement une réduction des opérations de surveillance et de maintenance,

- concernant les deuxième et troisième niveaux de la défense en profondeur, la faible pression du fluide de refroidissement du cœur (fluide primaire) dans la plupart des réacteurs de recherche permet d'éviter la mise en place de circuits d'injection de sécurité $^{175}$. Seul le réacteur Jules Horowitz dispose d'un circuit de ce type compte tenu de la densité de puissance dans le cœur et de la pression du

173. Les éléments qui suivent peuvent concerner aussi d'autres installations nucléaires, notamment les réacteurs de puissance pour lesquels une extension de leur durée d'exploitation est envisagée. Le niveau d'attention à porter à ces sujets est bien évidemment à ajuster, selon une approche graduée, en fonction des risques présentés par l'installation étudiée.

174. Cette préoccupation a notamment concerné le réacteur PHENIX, à l'occasion de la reprise de son fonctionnement au début des années 2000, après une longue période d'arrêt pour des travaux de jouvence et d'amélioration de la sûreté. Du fait de l'unicité de certains réacteurs de recherche, un maintien des connaissances et des compétences sur site paraît aussi indispensable sur les aspects liés aux études de conception, à la construction et aux essais de démarrage.

175. Même si les réacteurs de recherche disposent de circuits d'appoint d'eau ou de mise en communication de capacités d'eau. 
fluide primaire (une dizaine de bars à l'entrée du cœur). Pour beaucoup de réacteurs de recherche, leurs caractéristiques intrinsèques permettent un refroidissement par convection naturelle ;

- concernant le quatrième niveau de la défense en profondeur, pour un grand nombre de réacteurs de recherche, un accident de référence avec fusion de combustible a été retenu pour le dimensionnement - ou la vérification d'un dimensionnement approprié ${ }^{176}$ - de la piscine du réacteur, des superstructures du bâtiment du réacteur, des systèmes de ventilation et de filtration. Ce sujet est développé plus loin dans ce chapitre.

Par ailleurs, certains réacteurs de recherche ont été construits dans des zones qui se sont progressivement urbanisées, ce qui accroît l'attention à porter à la réduction des conséquences de situations accidentelles et à l'adéquation des plans d'urgence. Cela peut constituer une condition à leur poursuite d'exploitation.

En France, différents codes de conception et de construction peuvent être utilisés ou servir de référence (pour ceux qui visent formellement les réacteurs à eau sous pression) pour un projet de réacteur de recherche et de ses dispositifs associés, ou pour des modifications à apporter à un réacteur de recherche existant (nouveau dispositif expérimental, modifications apportées dans le cadre d'un réexamen de sûreté...). À cet égard, le RCC-MRx, élaboré par le CEA et des industriels, est appliqué aux matériels mécaniques du réacteur Jules Horowitz (voir le focus ci-après). Les RCC-E (règles de conception et de construction des équipements électriques et de contrôle commande des ilots nucléaires des réacteurs à eau sous pression), et RCC-CW (règles de conception et de construction du génie-civil des ilots nucléaires des réacteurs à eau sous pression) peuvent servir de référence pour les réacteurs de recherche.

\section{\#FOCUS}

\section{Des règles de conception et de construction codifiées pour les matériels mécaniques, applicables notamment aux réacteurs de recherche : le $\mathrm{RCC}_{-M R x^{177}}$}

Le « code » RCC-MRx réalise, depuis 2009, la fusion de deux documents :

- le RCC-MR développé à partir de 1985 pour les réacteurs à neutrons rapides (RNR) refroidis au sodium (réacteurs fonctionnant à températures élevées, jusqu'à plus de $500{ }^{\circ} \mathrm{C}$ en fonctionnement normal) ;

- le RCC-MX développé à partir de 1998 par le CEA, AREVA-TA et AREVA-NP pour les besoins spécifiques du projet de réacteur Jules

176. Eu égard aux conséquences radiologiques possibles d'un tel accident.

177. D'après le site internet de l'AFCEN. La version du RCC-MRx visée ici est celle de 2015, la plus récente à la date de la finalisation du présent ouvrage. 
Horowitz (réacteur et ses auxiliaires, dispositifs expérimentaux associés) et également utilisable pour la conception et la construction de matériels ou dispositifs pour des réacteurs de recherche en exploitation.

Le RCC-MRx élargit le domaine d'applications possibles aux réacteurs de fusion nucléaire (projet ITER par exemple). Il fournit des règles pour les composants mécaniques sollicités dans le domaine du fluage significatif (RNR) ou de l'irradiation significative (RNR, réacteurs de recherche et leurs dispositifs expérimentaux). Il fournit les caractéristiques mécaniques d'un panel étendu de matériaux (aciers, alliages 800 , alliages d'aluminium et de zirconium permettant de répondre aux besoins de transparence aux neutrons pour les réacteurs de recherche), des règles de dimensionnement des coques minces et des caissons, de nouveaux procédés de soudage (par faisceau d'électron, laser, diffusion...), etc.

La version de 2015 du RCC-MRx intègre le retour d'expérience consécutif à l'utilisation des éditions précédentes, en particulier dans le cadre des projets comme celui du réacteur Jules Horowitz; il s'agit en particulier du retour d'expérience sur le contrôle et les procédés de soudage des alliages d'aluminium.

Lors de son élaboration et de ses mises à jour, une attention particulière est portée à la cohérence du RCC-MRx avec les autres « référentiels » qui interagissent avec lui : RCC-M, textes officiels français, normes européennes et internationales.

Quelques-uns des sujets traités dans le RCC-MRx sont indiqués ci-après.

\begin{tabular}{|l} 
1. INTRODUCTION \\
- Domaine d'application du code \\
- \\
-
\end{tabular}




\begin{tabular}{|c|c|}
\hline & $\begin{array}{l}\text { CARACTERISTIQUES DES MATERIAUX (ANNEXE A3) ET DES } \\
\text { OINTS SOUDES (ANNEXE A9) }\end{array}$ \\
\hline & $\begin{array}{l}\text { METHODES DE CONTROLE } \\
\text { Essais mécaniques, physiques, chimiques } \\
\text { Examen par ultrasons } \\
\text { Examen par radiographie } \\
\text { Examen par ressuage } \\
\text { Méthodes de détection des fuites }\end{array}$ \\
\hline & $\begin{array}{l}\text { ROUDAGE } \\
\text { Qualification des produits d'apport } \\
\text { Qualification du mode opératoire de soudage } \\
\text { Qualification des soudeurs } \\
\text { Qualification technique des ateliers de fabrication } \\
\text { Réalisation des soudures « de production » } \\
\text { Rechargements durs par fusion sur les aciers } \\
\text { Essais mécaniques } \\
\text { Particularités liées au soudage des alliages d'aluminium et de zirconium } \\
\text {... }\end{array}$ \\
\hline & $\begin{array}{l}\text { FBRICATION } \\
\text { Procédés de marquage } \\
\text { Découpe et réparation sans soudage } \\
\text { Formage et tolérances dimensionnelles } \\
\text { Traitement de surface } \\
\text { Propreté } \\
\text { Assemblages mécaniques vissés ou brasés } \\
\text { Traitements thermiques } \\
\text {... }\end{array}$ \\
\hline
\end{tabular}

\subsection{2. Événements retenus pour la conception et la démonstration de la sûreté des réacteurs de recherche}

La conception d'un réacteur de recherche et la démonstration de la sûreté associée reposent notamment sur l'identification de tous les événements (défaillances internes, agressions internes ou externes) susceptibles d'affecter l'installation. Toutefois, en 
fonction de l'événement considéré, la fréquence estimée de l'événement est plus ou moins élevée. Par exemple, la rupture complète d'une tuyauterie est considérée comme moins probable qu'une fuite modérée de cette tuyauterie.

Pour ce qui concerne les événements correspondant à des défaillances internes propres à l'installation ou à des erreurs d'opérateur, cela a conduit, pour les réacteurs de recherche les plus anciens, à en retenir un petit nombre, ayant un caractère enveloppe et hiérarchisés globalement en trois grandes catégories, à savoir les événements normaux, les incidents et les accidents.

Pour les réacteurs de recherche plus récents ou lors des réexamens de sûreté des plus anciens, par similitude avec les pratiques adoptées pour les réacteurs de puissance, il est retenu un certain nombre (jusqu'à quelques dizaines) de « conditions de fonctionnement » enveloppes pour l'installation, classées dans quatre catégories en fonction de la fréquence d'occurrence estimée de la famille ${ }^{178}$ d'événements initiateurs qu'elles couvrent.

Le tableau 7.1, à la fin de ce paragraphe, présente les différentes catégories de conditions de fonctionnement avec, à titre d'illustration, quelques-unes des conditions de fonctionnement d'un réacteur à canaux neutroniques.

L'étude des différentes « conditions de fonctionnement " permet notamment de déterminer différents chargements thermomécaniques sur les matériels de l'installation, appelés " situations de dimensionnement ", également répartis en catégories. Le dimensionnement des matériels consiste à vérifier ou à faire en sorte que, par des choix de conception pour ces matériels, les critères de codes de conception et de construction (par exemple le RCC-MRx), choisis en fonction de la catégorie de la « situation de dimensionnement " étudiée, sont satisfaits. Les critères choisis dépendent également d'autres considérations : l'importance pour la sûreté du matériel étudié (son classement de sûreté) et son rôle (actif ou passif) dans la situation considérée.

Des « limites de service » pour le combustible du cœur d'un réacteur de recherche sont généralement associées aux différentes catégories de « conditions de fonctionnement ॥. Il doit être en particulier visé que les conditions de fonctionnement de $1^{\text {ère }}$ et de $2^{\mathrm{e}}$ catégories (conditions normales de fonctionnement et transitoires fréquents) ne conduisent $\mathrm{ni}$ à la perte d'étanchéité des gaines ni à la fusion de combustible.

La démarche se référant à des catégories de « conditions de fonctionnement » n'a été véritablement mise en œuvre pour les réacteurs de recherche qu'à partir des années 2000. Le premier cas d'application concret a concerné le réacteur CABRI dans le cadre de l'installation d'une boucle à eau sous pression (BEP) et d'une réévaluation de sûreté complète du réacteur. Le réacteur Jules Horowitz a ensuite fait l'objet d'une

178. Événements liés à la réactivité neutronique du cœur, événements liés au refroidissement du réacteur, etc. Le tableau 3.3 dans la première partie du présent ouvrage fait apparaître des événements regroupés par familles. 
telle démarche, mise en œuvre au stade de la demande d'autorisation de création correspondante. Cette démarche a aussi été suivie pour la jouvence du réacteur CABRI.

Une spécificité des réacteurs de recherche concerne l'établissement des « conditions de fonctionnement » de première catégorie, dites normales, car, outre le fonctionnement stable du réacteur ainsi que les transitoires normaux de démarrage et d'arrêt du réacteur, il convient de prendre en compte tous les transitoires (normaux) associés à la réalisation des expériences d'irradiation envisagées. Or il n'est pas toujours possible pour un concepteur ou un exploitant de disposer, au moment des études de conception d'un réacteur de recherche, d'un programme suffisamment détaillé des expériences qui y seront réalisées. La démarche adoptée consiste alors à définir un domaine suffisamment enveloppe de transitoires normaux pour les expériences envisageables, en termes d'évolutions temporelles, pour le réacteur, de températures des fluides dans les différents circuits du réacteur, de pressions dans ces circuits, de débits des fluides, de flux neutronique dans le cœur, etc.

En outre, pour l'établissement de la liste des incidents et des accidents d'un réacteur de recherche, ou des " conditions de fonctionnement » de deuxième, de troisième et de quatrième catégories, les possibilités de défaillances ou d'erreurs lors du déroulement des expériences qui pourraient avoir des conséquences sur le réacteur lui-même sont à prendre en compte, ce qui peut poser quelques difficultés si les différents types de dispositifs expérimentaux envisagés pour le réacteur ne sont pas encore totalement définis.

Le sujet des interactions entre un réacteur de recherche et les dispositifs expérimentaux qui lui sont associés a fait l'objet de nombreux échanges techniques dans les années 2000 entre le CEA, l'ASN et l'IRSN à l'occasion de l'établissement, par le CEA et pour son propre usage, d'un guide de conception des dispositifs expérimentaux. L'établissement d'un tel guide était en outre, pour l'ASN, une condition à l'instauration d'un système d'autorisations internes ${ }^{179}$ au sein du CEA. L'objectif de ce guide était de formaliser quelques grands principes et une démarche d'analyse à adopter pour la conception de dispositifs expérimentaux tout particulièrement, cela en fonction de différents éléments : risques potentiels présentés par le dispositif, nombre et la robustesse des « barrières » séparant la zone d'essai au sein de ce dispositif et le cœur du réacteur ${ }^{180}$, systèmes d'accrochage et d'anti-envol ${ }^{181}$ du dispositif.

Dans ce guide, tel qu'il a été mis au point en janvier 2007, la démarche d'analyse préconisée s'appuie sur une approche utilisant le concept de « lignes de défense ${ }^{182}$ ». Le

179. Notion précisée au paragraphe 6.4 .

180. Sachant que l'une des contraintes est d'obtenir une séparation suffisamment transparente aux neutrons entre le cœur du réacteur et la zone d'essai.

181. Cela concerne, par exemple, les dispositifs expérimentaux qui sont de nature, en cas de déplacement vertical intempestif voire d'éjection, à apporter un surcroît de réactivité au cœur. Un tel événement peut être naturellement la conséquence de phénomènes énergétiques pouvant survenir - voire même être recherchés - au sein du dispositif, selon les objectifs d'une expérience menée avec ce dispositif.

182. Voir plus loin le nota 189 au paragraphe 7.1.2. 
guide définit en outre les règles permettant au CEA d'autoriser en interne la mise en place d'un nouveau dispositif expérimental dans un de ses réacteurs de recherche.

De façon générale, le guide a permis d'améliorer et de faciliter les analyses et les expertises de sûreté des dispositifs expérimentaux. Si l'ASN ne s'est pas formellement prononcée sur ce guide - qui néanmoins avait fait l'objet d'échanges avec elle (notamment sous la forme de réunions de travail) -, elle en a fait état dans son autorisation au CEA de mettre en place son système d'autorisations internes, notamment l'organisation associée.

Ce qui vient d'être présenté concerne seule la sûreté de fonctionnement du réacteur (avec ses dispositifs expérimentaux). Il ne faut pas oublier que l'environnement du réacteur peut être une source d'" agressions » susceptibles d'affecter les installations. En fait, deux types d'agressions sont considérés : les agressions internes, qui ont leur origine à l'intérieur de celles-ci, telles qu'un incendie par exemple, et les agressions externes, telles qu'un séisme ou l'impact d'un avion. Toutes les sources possibles d'agressions doivent être identifiées et traitées pour la conception du réacteur et pour la démonstration de sûreté associée. En particulier, les agressions externes déterminent également des " situations de dimensionnement » pour les équipements, plus souvent dénommées " cas de charge », l'objectif général retenu à l'égard des agressions étant qu'elles ne compromettent pas la disponibilité des fonctions fondamentales de sûreté, en dépit des effets directs et indirects de ces agressions.

Ainsi, comme pour les réacteurs à eau sous pression du parc électronucléaire, l'approche " cas de charge » a été complétée (lors de réexamens de sûreté ou pour les nouveaux projets de réacteurs de recherche) par la mise en œuvre d'une approche " séisme-événement », consistant à prendre en compte le fait qu'un séisme peut être à l'origine d'autres agressions, internes, par défaillance d'équipements non dimensionnés aux séismes. Il est à noter que, dans le principe, une telle approche peut être pertinente pour d'autres agressions que le séisme. L'approche conduit à :

- identifier les équipements non dimensionnés aux séismes ;

- étudier les conséquences de leur défaillance en cas de séisme, dans le but notamment de savoir si elles peuvent mettre en cause les exigences fonctionnelles d'équipements dimensionnés aux séismes et permettant l'accomplissement des fonctions fondamentales de sûreté ;

- si cela est le cas, décider des éventuelles dispositions à prendre : renforcer des équipements non dimensionnés aux séismes, protéger des équipements importants pouvant être agressés.

Par ailleurs, il est postulé qu'un séisme entraîne la perte des sources électriques externes $^{183}$ (perte du réseau).

183. MDT ou MDTE : manque de tension externe. 
Comme pour les réacteurs de puissance du parc électronucléaire, un domaine complémentaire de conditions de fonctionnement correspondant à des défaillances multiples ou à des cumuls d'événements, qui peuvent être de très faible probabilité, est dorénavant pris en compte pour la conception et la démonstration de sûreté des réacteurs de recherche. Par exemple, une perte totale des alimentations électriques ${ }^{184}$, un séisme majoré de sécurité ${ }^{185}$ supposé survenir alors qu'une charge (lourde) est en cours de manutention dans le bâtiment du réacteur - avec comme exigence la non-chute de cette charge -, etc. À cet égard, le concepteur du réacteur Jules Horowitz utilise l'expression "situations de limitation du risque "(SLR), ces situations comprenant notamment des « accidents graves maîtrisés » (AGM) relevant du quatrième niveau de la défense en profondeur.

Enfin, comme pour les réacteurs du parc électronucléaire, une attention particulière doit être portée aux événements " exclus » lors de la constitution de la liste des « conditions de fonctionnement ». Ces événements ne faisant pas l'objet de dispositions spécifiques pour en limiter les conséquences (elles peuvent se révéler irréalisables), leur « exclusion » doit être justifiée en montrant que soit leur survenue est impossible physiquement, soit leur probabilité est très faible, cela avec un haut niveau de confiance. Dans ce second cas, une analyse au cas par cas est souhaitable, un seuil de coupure générique en termes de probabilité n'apparaissant pas pertinent ${ }^{186}$. La prévention des événements « exclus » suppose des dispositions renforcées, en termes de conception, de construction et d'inspection en service, par rapport à celles adoptées pour la prévention des événements dont la survenue n'est pas « exclue ».

La démarche développée ci-dessus est progressivement appliquée aux réacteurs de recherche anciens, à l'occasion de leurs réexamens de sûreté : outre le cas du réacteur CABRI évoqué plus haut, on peut citer ici le cas du réacteur ORPHEE à Saclay et celui du RHF à Grenoble.

Ainsi, parmi les événements étudiés pour les réacteurs de recherche, certains sont semblables à ceux retenus pour les réacteurs du parc électronucléaire, tels que la brèche, voire la rupture complète d'une tuyauterie du circuit primaire, le retrait intempestif d'un élément absorbant hors de la zone du cœur ou encore la perte partielle, voire complète des alimentations électriques. Certains autres événements sont spécifiques des réacteurs de recherche compte tenu des expériences qui y sont menées ou envisagées.

Les analyses de sûreté - et les expertises menées par l'IRSN - effectuées dans le cadre des travaux de mise à niveau du réacteur $C A B R I$, avec l'installation dans ce réacteur de la boucle à eau sous pression en remplacement de la boucle en sodium, ont par ailleurs amené à adopter des règles et des pratiques utilisées pour les réacteur à eau sous pression - la boucle, ses systèmes associés et son caisson de confinement constituant

184. Perte des alimentations électriques externes cumulée à la perte des groupes électrogènes principaux (MDTG : manque de tension généralisé).

185. Voir le paragraphe 7.4.2.

186. Voir à ce sujet les « directives techniques pour la conception et la construction de la prochaine génération de tranches nucléaires à eau pressurisée », établies par le GPR et les groupes d'experts allemands au mois d'octobre 2000 et utilisées pour le projet de réacteur EPR. 
en eux-mêmes le circuit primaire, les systèmes associés et l'enceinte d'un REP. Quelques aspects sont donnés ci-après à titre illustratif :

- si les « conditions de fonctionnement » associées au réacteur proprement dit ont été définies en s'inspirant fortement des événements retenus pour les réacteurs de recherche de type piscine, la définition des " conditions de fonctionnement 》 associées à la boucle s'est naturellement inspirée des « conditions de fonctionnement » retenues pour les réacteurs à eau sous pression ;

- le caisson de la boucle à eau a été dimensionné pour résister à une " pression de calcul » correspondant à celle qui serait atteinte dans le cas d'une brèche dans les parois de la boucle - partie équivalente au circuit primaire principal d'un REP correspondant à l'accident de perte de réfrigérant primaire étudié pour un tel réacteur $^{187}$; les prescriptions relatives aux enceintes de confinement métalliques dans le code de conception et de construction ASME, largement utilisé de par le monde pour les réacteurs à eau légère, ont été prises comme référence dans ce domaine ;

- le risque de rupture (complète) de la partie en réacteur de la boucle (« cellule en pile ») pouvant avoir des conséquences importantes sur le cœur nourricier (la dépressurisation brutale de l'eau de la boucle pouvant compacter les assemblages du cœur nourricier et empêcher la chute des barres absorbantes), il devait être rendu suffisamment improbable. Les soupapes de sécurité prévues sur le circuit primaire de la boucle participent bien évidemment à la prévention d'un tel événement ; elles devaient en tout état de cause respecter la règlementation visant les organes de sécurité (soupapes) pour les appareils à pression ;

- la boucle à eau sous pression a été soumise aux exigences de la règlementation des appareils à pression (arrêté ESPN).

Ces cas d'utilisation combinée des règles et pratiques en usage pour les réacteurs de recherche et pour les réacteurs à eau sous pression n'ont pas fait apparaître de difficultés, cela traduisant une bonne cohérence et compatibilité des démarches.

Enfin, comme cela a été indiqué plus haut, des études probabilistes de sûreté peuvent, dans certains cas, apporter des éclairages utiles en complément de la démarche déterministe, dans le cadre de nouveaux projets, de modifications importantes ou de réévaluations de sûreté de réacteurs de recherche ${ }^{188}$. Par exemple, des études probabilistes ciblées peuvent aider à orienter certains choix de conception : une étude probabiliste des défaillances possibles de l'évacuation de la puissance résiduelle peut permettre de choisir des options de conception en termes d'architecture générale des circuits de refroidissement, de redondance ou de diversification d'équipements. Les études probabilistes de sûreté peuvent aussi permettre de conforter ou d'amender le

187. Il s'agit en l'occurrence d'une rupture complète doublement débattue, à l'intérieur du caisson, de la tuyauterie primaire de la boucle (dite 2A, A désignant la section de passage du fluide dans la tuyauterie), cumulée à la rupture d'une tuyauterie d'air comprimé, cette deuxième rupture pouvant être une conséquence de la première.

188. Voir aussi le paragraphe 3.4.3. En 2010, l'IRSN a réalisé une étude de faisabilité d'une EPS de niveau 1 pour le réacteur Jules Horowitz : voir la communication faite au congrès PSAM 2010, 10th International Probabilistic Safety Assessment, Feasibility study to develop a PSA for the Jules Horowitz research reactor, Laborde A., Georgescu G., Cochemé F., Lanore J.-M. 
classement de sûreté des équipements. Par ailleurs, en l'absence de modèles d'études probabilistes de sûreté, une approche simplifiée par « lignes de défense ${ }^{189}$ " peut apporter aussi des éclairages utiles, comme cela a été le cas au début des années 2000 dans le cadre d'une réévaluation de sûreté du RHF à Grenoble.

\subsubsection{Accidents de référence}

Comme cela a été indiqué plus haut, dès la conception des premiers réacteurs de recherche, il y a environ une cinquantaine d'années, la possibilité d'incidents et d'accidents a été considérée. Des incidents et des accidents ont dès lors été étudiés, et tout particulièrement des accidents dits (en France) de référence. Ces accidents considérés comme les plus graves envisageables permettent d'apprécier le caractère acceptable des dispositions techniques et organisationnelles retenues pour assurer la sûreté de l'installation considérée.

Ces accidents de référence ont été définis en tenant compte des spécificités des réacteurs et en considérant généralement des défaillances de plusieurs systèmes ou des erreurs humaines, conduisant ou susceptibles de conduire à un endommagement d'éléments combustibles voire du cœur du réacteur.

Pour les réacteurs de recherche refroidis par de l'eau et utilisant du combustible à base d'uranium et d'aluminium (avec gaine en aluminium), l'accident de type BORAX - du nom d'une installation américaine dans laquelle furent réalisés des essais sur ce type d'accident - a été retenue en France. Ce type d'accident est représentatif des risques qui pourraient résulter d'une insertion brutale d'une importante réactivité dans le cœur, à savoir principalement la fusion d'une partie voire de la totalité du cœur accompagnée éventuellement d'une « explosion de vapeur » dans la piscine.

Une insertion de réactivité entraîne un emballement de la réaction en chaîne, qui peut être limité par les effets qu'il produit car l'augmentation des températures du combustible et de l'eau a un effet négatif sur la réactivité (contre-réactions neutroniques). Néanmoins, si l'insertion de réactivité est trop rapide et trop importante, les contreréactions ne sont pas suffisantes pour éviter un endommagement du combustible. Dans le cas de l'accident de type BORAX, qui concerne les réacteurs utilisant du combustible à base d'uranium et d'aluminium, les températures du combustible et des gaines augmentent jusqu'à atteindre rapidement leur température de fusion $\left(660^{\circ} \mathrm{C}^{190}\right)$. Le combustible peut alors se disperser dans l'eau, qui est restée relativement froide compte tenu de la cinétique de l'accident, et provoquer une explosion de vapeur du fait du transfert brutal d'énergie des matériaux fondus à l'eau.

Compte tenu des conséquences destructrices d'un tel accident, l'accent est d'abord mis sur la prévention des défaillances pouvant y conduire. L'accident est néanmoins supposé pouvoir se produire. Sa cinétique étant trop rapide pour qu'un arrêt d'urgence puisse être suffisamment efficace, des dispositions sont prises pour en limiter les conséquences. Elles reposent principalement sur la capacité de la piscine à résister à l'éventuelle explosion de vapeur pour maintenir le cœur fondu sous eau (l'eau assurant le

189. Cette approche est notamment présentée dans la communication de M. Lavérie (chef du SCSIN de 1986 à 1993) à une conférence tenue en 1982 à Lyon sur les réacteurs à neutrons rapides refroidis par un métal liquide (cf. Proceeding of the LMFBR Safety Topical Meeting, Lyon (1982), p. I-335). 
refroidissement et une protection biologique), ainsi que sur la capacité du bâtiment du réacteur à résister à l'accident et à confiner les produits radioactifs relâchés dans ce bâtiment. En dehors de la France, ce type d'accident a été pris en compte de façon complète (interaction combustible-eau) pour le réacteur BR2 du centre de $\mathrm{Mol}$ en Belgique.

Pour les réacteurs OSIRIS et ORPHEE ainsi que pour le RHF, un accident de fusion à l'air d'un élément combustible en cours de manutention dans le bâtiment du réacteur (pouvant être occasionné par des défaillances au cours d'une telle manutention) a également été retenu. Par rapport à un accident de fusion de combustible sous eau - tel que l'accident de type BORAX -, un accident de fusion à l'air conduit à davantage de radionucléides émis dans le bâtiment du réacteur, car dans ce cas il n'y a pas d'eau pour piéger les produits de fission. La possibilité d'une fusion du cœur du réacteur à l'air a été retenue dès la conception du réacteur RHF.

Nous reviendrons au chapitre 8 sur les accidents de référence retenus pour les réacteurs de recherche français et sur l'accident de type BORAX en particulier.

\subsubsection{Déclinaison des objectifs généraux de sûreté}

Les objectifs généraux de sûreté, exprimés de façon qualitative, ont été évoqués au paragraphe 7.1. Toutefois, comme pour les réacteurs électronucléaires, les concepteurs et les exploitants de réacteurs de recherche peuvent être amenés à retenir des objectifs généraux de sûreté exprimés de façon quantitative en termes de conséquences radiologiques " acceptables » sur l'homme et l'environnement, sous la forme de "valeurs repères » en termes de doses, pour les différentes catégories de " conditions de fonctionnement » et pour les conditions du domaine complémentaire. Si cela permet de structurer les études de conception et d'apprécier les choix opérés à ce stade, en cohérence avec le diagramme schématique de Farmer représenté ci-après, ces « valeurs repères » ne peuvent en aucun cas constituer des critères d'acceptabilité, les conséquences radiologiques devant être en tout état de cause rendues aussi faibles que raisonnablement possible en tenant compte des facteurs économiques et sociaux (principe d'optimisation).

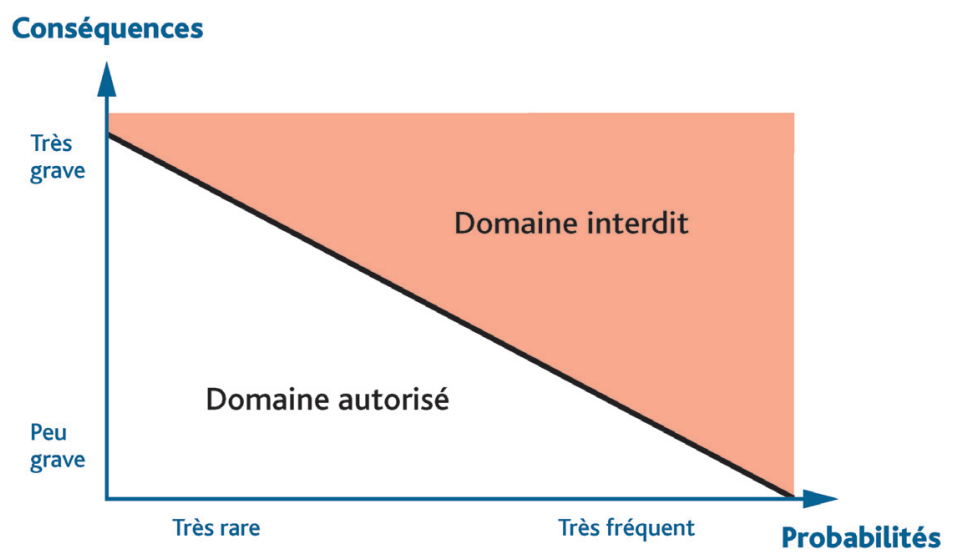

Figure 7.1. Représentation symbolique de Farmer de la relation entre probabilité et conséquences. 
Il faut rappeler que l'appréciation de la sûreté repose d'abord sur la bonne déclinaison du principe de défense en profondeur et non sur la simple comparaison des conséquences radiologiques calculées à des valeurs préétablies. En particulier, le calcul des conséquences radiologiques individuelles ne saurait traduire l'importance de ces conséquences, qui dépend aussi non seulement du nombre de personnes concernées (qui peut être important pour les réacteurs de recherche situés dans des zones fortement urbanisées) mais aussi de la contamination en termes d'étendue et de durée pouvant résulter d'un accident.

Par ailleurs, les objectifs généraux de sûreté peuvent être déclinés par les concepteurs ou exploitants en critères « relais » ou critères « de découplage » utilisés pour l'étude des conditions de fonctionnement et de l'adéquation des dispositions permettant d'en limiter leurs conséquences (pourcentage de ruptures de gaines d'éléments combustibles, pourcentage de combustible fondu, etc.).

\subsubsection{L'approche graduée en France}

L'arrêté du 7 février 2012 qui fixe les règles générales applicables à la conception, à la construction, au fonctionnement, à la mise à l'arrêt définitif, au démantèlement, à l'entretien et à la surveillance des installations nucléaires de base souligne que « leur application repose sur une approche proportionnée à l'importance des risques ou inconvénients présentés par l'installation ". Cet arrêté évoque plus particulièrement cette " approche proportionnée » pour le nombre et l'efficacité des «barrières » de confinement - aspect qui concerne tout particulièrement la conception des réacteurs de recherche, le nombre de leurs « barrières » pouvant varier d'un réacteur à l'autre -, la qualification des éléments importants de l'installation, la fréquence des exercices de gestion de situations d'urgence ou encore la surveillance des intervenants extérieurs.

Il est aussi à souligner que l'utilisation d'une démarche déterministe fondée sur la définition et l'étude de « conditions de fonctionnement » issus d'événements initiateurs internes, d'agressions internes (liées au réacteur lui-même) et d'agressions externes (liées au site du réacteur) pour la conception, la démonstration de sûreté ou encore les réévaluations de sûreté d'un réacteur de recherche conduit de fait à des dispositions de sûreté adaptées au réacteur de recherche étudié et au site sur lequel il est implanté et proportionnées aux risques qu'ils présentent.

Il est également à noter que le classement de sûreté des équipements au sein d'une même installation nucléaire conduit à proportionner un certain nombre d'exigences les concernant à leur importance pour la sûreté (coefficients de sécurité pour leur dimensionnement, types de soudures autorisées ou non par les codes de conception et de construction, étendue et nature des contrôles de fin de fabrication, des contrôles en service...). 
Tableau 7.1. Conditions de fonctionnement : classement en catégories et quelques exemples donnés à titre illustratif pour un réacteur de type piscine.

\begin{tabular}{|c|c|c|}
\hline $\begin{array}{l}\text { Catégories de } \\
\text { conditions de } \\
\text { fonctionnement }\end{array}$ & $\begin{array}{l}\text { Ordre de grandeur de la } \\
\text { fréquence annuelle par } \\
\text { réacteur (et borne } \\
\text { supérieure exprimée en } \\
\text { probabilité) }\end{array}$ & $\begin{array}{c}\text { Exemples de conditions de } \\
\text { fonctionnement pour un réacteur de type } \\
\text { piscine, avec canaux neutroniques } \\
\text { (indiqué par }{ }^{*} \text { ), utilisant de l'eau lourde } \\
\text { (indiqué par }{ }^{* *} \text { ) - Hors événements à } \\
\text { prendre en compte pour les hottes de } \\
\text { transfert d'éléments combustibles (perte } \\
\text { de refroidissement...) }\end{array}$ \\
\hline $\begin{array}{l}\text { CATEGORIE } 1 \\
\text { Conditions } \\
\text { normales } \\
\text { d'exploitation }\end{array}$ & $\begin{array}{l}\text { Nombre d'occurrences } \\
\text { défini selon le programme } \\
\text { d'exploitation } \\
(P=1)\end{array}$ & $\begin{array}{l}\text { - États stables et transitoires normaux } \\
\text { d'exploitation (expériences d'irradiation } \\
\text { comprises) }\end{array}$ \\
\hline $\begin{array}{l}\text { CATEGORIE } 2 \\
\text { Incidents mineurs } \\
\text { mais fréquents }\end{array}$ & $\begin{array}{l}\text { Jusqu'à quelques } \\
\text { occurrences par an } \\
\qquad(P<1)\end{array}$ & $\begin{array}{l}\text { - Perte d'étanchéité de la gaine de crayons } \\
\text { ou de plaques combustibles } \\
\text { - Fuite ou brèche primaire de petit diamètre } \\
\text { équivalent (par exemple } \phi<10 \mathrm{~mm} \text { ) } \\
\text { - Perte partielle de débit primaire } \\
\text { - Perte totale de débit secondaire } \\
\text { - Fuite affectant un échangeur de chaleur } \\
\text { entre eau légère et eau lourde }\left(^{* *}\right) \\
\text { - Arrêt d'une pompe d'un circuit de refroi- } \\
\text { dissement à l'arrêt } \\
\text { - Perte d'étanchéité de l'enveloppe d'un } \\
\text { doigt de gant conduisant à une entrée } \\
\text { d'eau lourde dans le doigt de gant (*) }\left(^{* *} \text { ) }\right. \\
\text { - Fuite ou brèche affectant les dispositifs de } \\
\text { rejet d'effluents } \\
\text { - Perte de courte durée (par exemple } \\
<1 \text { heure) des alimentations électriques } \\
\text { externes }\end{array}$ \\
\hline $\begin{array}{l}\text { CATEGORIE } 3 \\
\text { Accidents peu } \\
\text { probables }\end{array}$ & $\begin{array}{c}<10^{-2} \\
\left(P<10^{-2}\right)\end{array}$ & $\begin{array}{l}\text { - Sortie ou retrait intempestif d'un élément } \\
\text { absorbant (éventuellement en cat. } 2 \text { ) } \\
\text { - Brèche primaire de diamètre équivalent } \\
\text { « intermédiaire » (par exemple } \\
10 \mathrm{~mm} \leq \phi<100 \mathrm{~mm} \text { ) } \\
\text { - Brèche d'un doigt de gant en aluminium } \\
\text { standard (*) } \\
\text { - Rupture d'une manchette d'eau lourde }\left(^{* *}\right) \\
\text { - Bouchage d'un canal de refroidissement } \\
\text { d'un élément combustible en cœur } \\
\text { - Bouchage de plusieurs canaux d'un élément } \\
\text { combustible en zone d'entreposage } \\
\text { - Chute, dans l'installation, d'un emballage } \\
\text { de transport contenant des éléments } \\
\text { combustibles } \\
\text {-... }\end{array}$ \\
\hline
\end{tabular}




\begin{tabular}{|c|c|c|}
\hline $\begin{array}{l}\text { CATEGORIE } 4 \\
\text { Accidents } \\
\text { importants mais } \\
\text { hypothétiques }\end{array}$ & $\begin{array}{c}<10^{-4} \\
\left(P<10^{-4}\right)\end{array}$ & $\begin{array}{l}\text { - Éjection d'un élément absorbant (pouvant } \\
\text { mener à une fusion dans le cœur) - éven- } \\
\text { tuellement étudiée comme condition de } \\
\text { fonctionnement complémentaire si cela } \\
\text { suppose de multiples défaillances } \\
\text { - Rupture du bloc-pile en piscine (entraî- } \\
\text { nant des transferts réciproques entre eau } \\
\text { légère et eau lourde) (**) } \\
\text { - Brèche primaire de gros diamètre équiva- } \\
\text { lent (par exemple } \phi \geq 100 \mathrm{~mm} \text { ) } \\
\text { - Brèche d'un doigt de gant en Zircaloy } \\
\text { - Bipasse du refroidissement du cœur }{ }^{191} \text { du } \\
\text { réacteur (pouvant mener à une fusion } \\
\text { dans le cœur) } \\
\text { - Rupture guillotine complètement débat- } \\
\text { tue, en piscine, d'un collecteur }{ }^{192} \text { d'entrée } \\
\text { d'eau dans le cœur } \\
\text { - En cellule chaude, découpe accidentelle } \\
\text { dans un élément combustible } \\
\text { - ... }\end{array}$ \\
\hline $\begin{array}{l}\text { Domaine dit du } \\
\text { hors } \\
\text { dimensionnement }\end{array}$ & $<10^{-6}, 10^{-7}$ par famille & $\begin{array}{l}\text { Conditions de fonctionnement complémentai- } \\
\text { res (certaines pouvant mener à une fusion dans } \\
\text { le cœur ou du cœeur, sous eau, ou à l'air en cas } \\
\text { de dénoyage d'éléments combustibles) } \\
\text { - Sortie intempestive d'un élément absor- } \\
\text { bant avec défaillance du système de pro- } \\
\text { tection du réacteur } \\
\text { - Rupture du circuit principal d'eau lourde } \\
\text { avec défaillance du clapet casse-siphon }\left(^{*}\right) \\
\text { - Perte de débit secondaire avec défaillance } \\
\text { du système de protection } \\
\text { - Rupture totale d'un doigt de gant avec } \\
\text { défaillance de l'isolement (« fenêtre } » \text { et } \\
\text { vanne de sécurité, ou vanne de sécurité } \\
\left.\text { amont et vanne de sécurité aval }{ }^{194}\right)(*) \\
\text { - Perte totale des alimentations électriques } \\
\text { externes et de premier secours } \\
\text { - Perte des alimentations électriques exter- } \\
\text { nes et défaillance du système de protec- } \\
\text { tion du réacteur } \\
\text { - Accident de type BORAX (accident de } \\
\text { réactivité) } \\
\text { - Perte de tout l'inventaire en eau (lourde } \\
\text { et légère) du réacteur (**) }\end{array}$ \\
\hline
\end{tabular}

191. Par exemple, dans le cas du RHF, une brèche de la « cheminée », structure située au-dessus du caisson du cœur.

192. Cas du RHF : voir la figure 5.12.

193. Dans le cas du RHF, cela se traduirait par une entrée d'eau légère dans l'élément combustible en cœur (à la place de l'eau lourde).

194. Voir la description de ces éléments au paragraphe 7.3.2. 


\begin{tabular}{|c|c|c|c|}
\hline & & & $\begin{array}{l}\text { Autres accidents étudiés, voire exclus par } \\
\text { des dispositions de prévention } \\
\text { - Dénoyage des éléments combustibles } \\
\text { entreposés } \\
\text { - Rupture des deux enceintes d'une «source } \\
\text { chaude » ou d'une « source froide » }\left(^{*}\right) \\
\text { - Explosion dans un doigt de gant de } \\
\text { " source froide ", conduisant à un } \\
\text { endommagement interne du bloc-pile }\left(^{*}\right) \\
\text { - Fusion du cœur avec défaillance totale du } \\
\text { confinement } \\
\text {-... }\end{array}$ \\
\hline
\end{tabular}

\subsection{Quelques spécificités de sûreté des réacteurs de recherche}

\subsubsection{Puissances volumiques, combustibles utilisés et caractéristiques neutroniques des cœurs}

Malgré une puissance globale largement inférieure, la puissance volumique dégagée par un réacteur de recherche est souvent, compte tenu de la taille du cœur, supérieure à celle d'un réacteur de puissance, afin d'obtenir les flux neutroniques nécessaires aux expériences ou aux irradiations ${ }^{195}$. Le tableau 7.2 présente quelques valeurs de puissances volumiques dans les cœurs de différents types de réacteurs, y compris de réacteurs de puissance.

Tableau 7.2. Puissance volumique dans le cœur et température du fluide caloporteur pour différents types de réacteurs.

\begin{tabular}{|l|c|c|c|c|c|}
\hline & $\begin{array}{c}\text { Réacteur à } \\
\text { eau sous } \\
\text { pression }\end{array}$ & $\begin{array}{c}\text { Réacteur à } \\
\text { neutrons rapides } \\
\text { refroidi par du } \\
\text { sodium }\end{array}$ & OSIRIS & RHF & RJH \\
\hline Puissance volumique $(\mathrm{kW} / \mathrm{l})$ & $\sim 100$ & $\sim 300$ & $\sim 300$ & $\sim 1200$ & $\sim 600$ \\
\hline $\begin{array}{l}\text { Température du fluide } \\
\text { caloporteur à l'entrée et à la } \\
\text { sortie du cœur }\left({ }^{\circ} \mathrm{C}\right)\end{array}$ & $286-323^{(*)}$ & $350-550$ & $38-48$ & $30-48$ & $25-36$ \\
\hline
\end{tabular}

${ }^{(*)}$ Ces valeurs correspondent précisément aux REP de 900 MWe de type Bugey.

195. La conception d'un réacteur de recherche doit rechercher le meilleur compromis entre plusieurs impératifs contradictoires : définir un cœur compact pour atteindre des densités de fission élevées, assurer un volume expérimental suffisant pour implanter tous les dispositifs expérimentaux, extraire des densités de puissance importantes sans nuire aux performances neutroniques du cœur ni gêner son utilisation expérimentale ("Les réacteurs de recherche », Francis Merchie, L'Encyclopédie de l'énergie, 2015). 
L'importance de la puissance volumique dégagée dépend du combustible utilisé. Elle peut être élevée avec de l'uranium très fortement enrichi en isotope 235. Plusieurs types de combustibles sont utilisés dans les réacteurs de recherche selon leurs utilisations. Pour les maquettes critiques dont la souplesse d'utilisation est essentielle, les exploitants disposent en général d'un large inventaire d'éléments combustibles, sous forme de réglettes ou de plaquettes, permettant de constituer des cœurs « à la carte ». Pour ce type de réacteur, les assemblages qui composent le cœur du réacteur sont constitués par des opérations manuelles, dans l'installation elle-même.

Pour les réacteurs d'irradiation ou les réacteurs à " faisceaux sortis de neutrons ", dont la raison d'être principale est la production d'un flux de neutrons important, le bon refroidissement des éléments combustibles lors du fonctionnement du réacteur devient primordial. La conception des éléments combustibles est adaptée à cette fin : par exemple, l'utilisation de plaques cintrées (figure 7.2) confèrent à celles-ci une grande raideur et permet d'assurer dans de multiples conditions dégradées ou d'agressions (séisme...) un maintien de l'espacement souhaité entre les plaques.

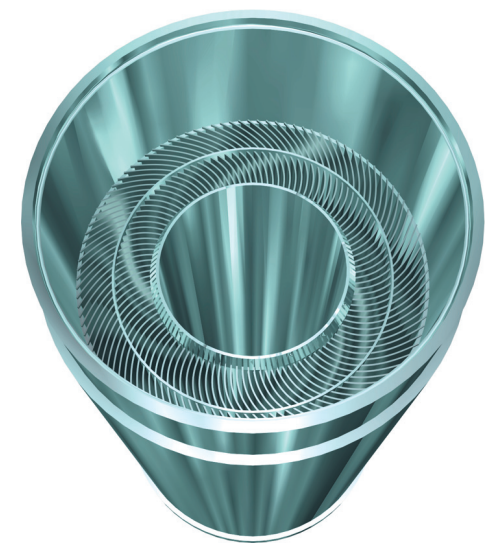

Figure 7.2. Élément combustible constitué de plaques cintrées utilisé dans le réacteur FRM-II de Garching en Allemagne. (c) FRMII Technical University of Munich.

Comme cela est indiqué au chapitre 2, dans le contexte international de lutte contre le risque de prolifération des armes nucléaires, l'approvisionnement en combustible fortement enrichi en isotope 235 de l'uranium pose problème. Aussi, un certain nombre de réacteurs de recherche ont fait l'objet d'une " conversion » à l'utilisation d'un combustible " siliciure », utilisant un alliage de formule $\mathrm{U}_{3} \mathrm{Si}_{2}$ et permettant de réduire l'enrichissement en uranium 235 à une valeur n'excédant pas $20 \%$, tout en conservant les potentialités et les capacités de ces réacteurs. Toutefois, cette " conversion » peut poser des difficultés pour certains réacteurs de recherche, compte tenu de certaines contraintes (dimensionnelles, etc.) rendant difficile des modifications, notamment de leur cœur. Pour compenser la perte sur l'enrichissement de l'uranium en isotope 235, de 
nouveaux alliages de plus grande densité ${ }^{196}$ en uranium sont étudiés, mais qui ne seraient pas exempt d'inconvénients (remplacement du combustible du cœur plus fréquent, diminution de l'efficacité des absorbants neutroniques...). Cette question est toujours d'actualité et elle est au cœur de la problématique du choix du combustible pour le réacteur Jules Horowitz ${ }^{197}$. Des recherches sont menées pour le développement et la qualification d'autres combustibles; le CEA et d'autres organismes ${ }^{198}$ étudient ainsi un combustible composé de grains d'uranium enrichi à $20 \%$ en isotope 235 et de molybdène, gainé par un alliage d'aluminium (dénommé UMo).

L'aluminium (ou des alliages d'aluminium comme l'AG3NET ou de zirconium) est largement utilisé dans les réacteurs de recherche : pour le combustible, pour le gainage du combustible, pour des structures internes et des équipements (comme des canaux neutroniques) de ces réacteurs. En effet, pour obtenir un bon rendement thermodynamique, les centrales nucléaires de puissance fonctionnent à des températures les plus élevées possibles alors que les réacteurs de recherche sont, sauf exception, des réacteurs " froids ». Cela autorise l'usage de ce type de matériaux, qui ont de bien meilleures caractéristiques neutroniques que l'acier (transparence aux neutrons), bien qu'ils puissent avoir, selon leur élaboration et leur traitement, de moins bonnes propriétés mécaniques (en termes de limite d'élasticité, d'allongement à rupture...).

En termes de neutronique ${ }^{199}$, les flux de neutrons thermiques utilisables pour les programmes expérimentaux doivent atteindre des valeurs de $1.10^{13}$ à plus de $1.10^{15}$ neutrons $/ \mathrm{cm}^{2}$.s. Le flux de neutrons qui règne dans un réacteur est composé de neutrons rapides directement issus des fissions, de neutrons thermiques après ralentissement dans le modérateur et enfin de neutrons intermédiaires en cours de ralentissement (le ralentissement s'effectue par chocs successifs des neutrons sur le noyau des atomes du modérateur). Selon que le réacteur sera bien modéré ou sous-modéré, le spectre des neutrons comportera une composante « rapide » plus ou moins importante ce qui peut être préjudiciable ou parfois souhaitable selon les objectifs des programmes expérimentaux.

Par ailleurs, la répartition spatiale des neutrons n'est pas uniforme et décroît du centre vers la périphérie du cœur en présentant des perturbations locales dues, entre autres, au mouvement d'un absorbant de contrôle et à la présence des dispositifs expérimentaux. Cela entraîne une répartition spatio-temporelle variable de la puissance calorifique générée et donc la présence de "points chauds » qui doivent être bien anticipés et traités du point de vue thermohydraulique pour éviter toute surchauffe du combustible au-delà des limites fixées pour empêcher sa dégradation. Cette préoccupation de sûreté sera illustrée au paragraphe 10.1.1, avec la fusion de quelques crayons combustibles découverte en 2004 dans le cœur nourricier du réacteur CABRI.

196. Le combustible « siliciure », de référence NUREG-1313, a une densité de 4,8 $\mathrm{gU} / \mathrm{cm}^{3}$. Il est visé d'atteindre, avec de nouveaux combustibles, une densité environ deux fois plus élevée.

197. « Les combustibles nucléaires », Monographie de la Direction de l'énergie nucléaire du CEA, 2008.

198. Les États-Unis (ANL), le Canada, la Russie, la Corée et l'Argentine apportent leur contribution à la qualification de ce nouveau combustible. Des expériences ont notamment été réalisées dans le réacteur OSIRIS ; les réacteurs HFR et BR2 sont aussi utilisés.

199. « Les réacteurs de recherche », Francis Merchie, L'Encyclopédie de l'énergie, 2015. 
Tableau 7.3. Quelques paramètres neutroniques relatifs au cœur de différents types de réacteurs.

\begin{tabular}{|c|c|c|c|c|c|}
\hline & $\begin{array}{l}\text { Réacteur à } \\
\text { eau sous } \\
\text { pression }\end{array}$ & $\begin{array}{l}\text { Réacteur à } \\
\text { neutrons } \\
\text { rapides } \\
\text { refroidi au } \\
\text { sodium : } \\
\text { PHENIX }^{(*)}\end{array}$ & OSIRIS & RHF & RJH \\
\hline $\begin{array}{l}\text { Flux } \\
\text { maximum en } \\
\text { neutrons } / \mathrm{cm}^{2} . \mathrm{s}\end{array}$ & 1 à $3.10^{13}$ & $4,5.10^{15}$ & $5,4.10^{14}$ & $1,9.10^{15(* *)}$ & $1,1.10^{15}$ \\
\hline $\begin{array}{l}\text { Effet } \\
\text { Doppler }\end{array}$ & $-3 \mathrm{pcm} /{ }^{\circ} \mathrm{C}$ & $-0,6 \mathrm{pcm} /{ }^{\circ} \mathrm{C}$ & $-3 \mathrm{pcm} /{ }^{\circ} \mathrm{C}$ & \multirow[b]{2}{*}{$\begin{array}{c}-17 \mathrm{pcm} /{ }^{\circ} \mathrm{C} \\
\text { (effet Doppler } \\
\text { compris) }\end{array}$} & $-2,5 \mathrm{pcm} /{ }^{\circ} \mathrm{C}$ \\
\hline $\begin{array}{l}\text { Effet du } \\
\text { modérateur }\end{array}$ & $\begin{array}{c}\mathrm{de}-10 \text { à } \\
-60 \mathrm{pcm} /{ }^{\circ} \mathrm{C}\end{array}$ & $\begin{array}{l}-0,06 \mathrm{pcm} /{ }^{\circ} \mathrm{C} \\
\text { globalement, } \\
\text { mais positif } \\
\text { dans certaines } \\
\text { zones du cœur }\end{array}$ & $-14 \mathrm{pcm} /{ }^{\circ} \mathrm{C}$ & & $-20 \mathrm{pcm} /{ }^{\circ} \mathrm{C}$ \\
\hline $\begin{array}{l}\text { Bêta effectif } \\
\text { (proportion } \\
\text { de neutrons } \\
\text { différés) }\end{array}$ & $\begin{array}{c}500 \text { à } 700 \\
\text { pcm }\end{array}$ & 325 pcm & 731 pcm & 713 pcm & 730 pcm \\
\hline
\end{tabular}

${ }^{(*)}$ Les valeurs indiquées sont relatives au réacteur PHENIX fonctionnant à la puissance maximale de 350 MWth (puissance retenue pour son fonctionnement de 2003 à 2010).

${ }^{(* *)}$ Cela correspond à la somme de $1,5.10^{15}$ neutrons $/ \mathrm{cm}^{2}$.s de neutrons thermiques, disponibles notamment au niveau du nez des doigts de gant et de $3,5 \cdot 10^{14}$ neutrons $/ \mathrm{cm}^{2}$.s de neutrons rapides au niveau de l'élément combustible constituant le cœur.

\subsubsection{Cadences d'utilisation}

Une autre particularité des réacteurs de recherche concerne leurs modalités d'utilisation. Les réacteurs d'irradiation ou à « faisceaux sortis de neutrons » fonctionnent par cycles. Durant un cycle, ils fonctionnent généralement à une puissance stable $24 \mathrm{~h} / 24 \mathrm{~h}$. À la fin du cycle, ils sont arrêtés pour recharger le cœur. Cela permet à ces réacteurs de fournir des neutrons de manière stable (faisceaux pour les expériences) et sur une durée importante (irradiation).

Dans le cas des maquettes critiques, les modalités de fonctionnement sont très différentes et très liées aux programmes expérimentaux à réaliser, qui peuvent durer de quelques mois à plusieurs années. Un tel programme débute par une période de construction du cœur expérimental, qui peut durer plusieurs mois. Elle est suivie de la phase expérimentale proprement dite durant laquelle le réacteur peut fonctionner quelques heures seulement par jour ou toute la journée ; il est généralement mis à l'arrêt chaque soir. Des changements de configuration du cœur peuvent aussi être réalisés au cours de cette phase pour répondre aux besoins expérimentaux.

Dans le cas des réacteurs dans lesquels sont réalisés des essais concernant directement la sûreté, le temps de préparation d'un ou d'une série d'essais peut être 
relativement long, comme par exemple lors du changement de la boucle d'essais du réacteur $C A B R I$ qui a nécessité des travaux qui ont duré plusieurs années. Le délai entre deux essais peut également être long par exemple parce que la manipulation d'un combustible d'essai dégradé nécessite des dispositions particulières. En revanche, la durée proprement dite d'un essai peut être très courte en comparaison des périodes de préparation et des périodes entre essais.

\subsubsection{Facteurs organisationnels et humains}

L'un des aspects des réacteurs de recherche est l'implication plus particulière, par rapport à d'autres installations nucléaires telles que les réacteurs électronucléaires, des hommes à la fois dans l'exploitation proprement dite et dans leur utilisation à des fins de recherche. Si, comme dans la plupart des installations nucléaires, les hommes réalisent de nombreuses opérations (pilotage d'un « procédé », rechargement du cœur, maintenance, contrôles...), dans le cas des réacteurs de recherche, ils peuvent être, fréquemment, impliqués dans la manipulation, à l'aide de perches de manutention, d'éléments combustibles, voire dans la constitution de ces éléments (comme cela est les cas pour certaines maquettes ou assemblages critiques).

Il convient de souligner aussi l'importance des opérations de manutention de dispositifs expérimentaux placés dans le cœur du réacteur ou à proximité de celui-ci.

De plus, dans de nombreux réacteurs de recherche, il existe deux populations différentes de travailleurs amenés à réaliser des actions pouvant affecter la sûreté : d'une part les équipes d'exploitation du réacteur chargées d'exploiter celui-ci dans le respect des limites de sûreté, d'autre part les expérimentateurs qui mettent en œuvre les dispositifs expérimentaux en vue d'obtenir des résultats de recherche. Ces derniers ne sont pas a priori aussi imprégnés des limites de sûreté (et de leurs motivations). Aussi, l'autorité du chef d'installation sur ces deux populations de travailleurs revêt un caractère primordial.

Par ailleurs, après une campagne d'essais, de nombreuses interventions humaines peuvent être nécessaires : démontage ou remontage de circuits préalablement aux essais suivants, nettoyage de composants... Les conséquences possibles en termes de sûreté et de radioprotection de ces interventions doivent être clairement appréhendées.

Enfin, les activités menées avec les réacteurs de recherche ont bien souvent un caractère évolutif et discontinu. Ces activités évoluent en effet en fonction des programmes de recherche à réaliser. Les conséquences de ces évolutions, même minimes, doivent être appréciées en termes de sûreté et de radioprotection. De plus, lorsque des évolutions importantes peuvent entraîner l'interruption de certaines activités pendant de longues périodes pour la réalisation des modifications matérielles correspondantes, l'effet de ces interruptions sur les compétences des agents doit être évalué.

L'appréciation de la maîtrise des risques associés aux activités humaines dans les réacteurs de recherche peut s'appuyer sur une démarche ${ }^{200}$ consistant notamment à :

- déterminer les activités « sensibles » pour la sûreté ; il s'agit des activités dont la mauvaise réalisation pourraient avoir des conséquences notables en termes de sûreté ;

200. Revue Contrôle $n^{\circ} 176$ de juillet 2007 : « Les facteurs organisationnels et humains et la sûreté des réacteurs d'expérimentation », F. Jeffroy et M.-L. Delaporte-Normier, IRSN, p 47. 
- recenser les dispositions prévues pour fiabiliser la réalisation de ces activités ;

- évaluer l'efficacité de ces dispositions.

Le caractère sensible d'une activité s'apprécie selon divers facteurs : les conséquences possibles d'une mauvaise réalisation, la complexité des tâches correspondantes, le caractère répétitif de sa réalisation, le nombre de travailleurs impliqués, les besoins de coordination...

À titre d'illustration, deux activités jugées particulièrement sensibles peuvent être citées pour les réacteurs de recherche français :

- pour la maquette MASURCA, la constitution d'éléments combustibles « à la carte » à partir de réglettes ou de plaquettes dans le magasin d'entreposage (risque de criticité en cas d'erreur) ;

- pour le réacteur ISIS utilisé pour des sessions successives de formation, le réglage fréquent des seuils de sécurité (risque de mauvaise protection du réacteur en cas de transitoire fortuit).

D’une manière générale, les expérimentations peuvent nécessiter la reprise de réglages du système de protection dont la fiabilité et la traçabilité doivent être assurées.

Si la grande majorité des activités dont la mauvaise réalisation peut avoir des conséquences notables en termes de sûreté ont été bien identifiées par les exploitants, d'autres activités pouvant avoir un effet sur la sûreté moins directement évident ou dont la sensibilité est liée à la complexité des opérations à réaliser peuvent encore nécessiter des approfondissements de la part des exploitants.

Il a été indiqué précédemment que les besoins de coordination entre différentes équipes fait généralement partie des aspects à prendre en compte pour apprécier le caractère sensible d'une activité. Il en est notamment ainsi pour la réalisation de certaines expérimentations nécessitant une préparation et une coordination particulièrement étroite entre le personnel d'exploitation et les expérimentateurs. Les essais du programme PhébusPF (voir le focus à la fin du chapitre 5) ont à cet égard fait l'objet d'une préparation approfondie pour réduire les risques de conflits d'intérêts dans leur déroulement : une instrumentation comprenant des dispositifs de mesure redondants et diversifiés et des procédures d'essais spécifiant des seuils d'arrêt prédéterminés ont été mises en œuvre. Le but était d'éviter d'arrêter trop tôt les essais, compte tenu des objectifs poursuivis, tout en assurant l'arrêt du réacteur et le maintien du confinement en cas de risque de dégradation excessive des « barrières » interposées entre le combustible d'essai et le cœur nourricier. Des procédures de conduite des essais ont été mises au point pour les opérateurs, spécifiant notamment la poursuite ou non des essais en fonction de l'état de l'instrumentation (dispositifs de mesure de température en service, défaillants ou saturés, etc.) et de limites prédéfinies. Ces dispositions ont été soumises à l'Autorité de sûreté nucléaire.

\subsection{Spécificités des réacteurs de recherche déclinées par fonction fondamentale de sûreté}

Dans ce paragraphe, sont présentées les principales spécificités techniques des réacteurs de recherche ayant une importance en termes de sûreté. 


\subsubsection{Maîtrise de la réactivité du cœur}

La réactivité du cœur est un paramètre (désigné par la lettre $\rho$ et exprimé en pcm pour cent mille) utilisé pour représenter la population (nombre) de neutrons dans un cœur et son évolution dans le temps. Lorsque cette population est stable, le réacteur est dit juste critique et sa réactivité est nulle $(\rho=0)$. Cette population est une résultante des neutrons générés par les fissions, de ceux émis de façon différée par certains produits de fission (neutrons dits retardés) et des neutrons qui fuient ou qui sont capturés (par exemple dans les matériaux absorbants). Dans le cœur d'un réacteur, la maîtrise de la réactivité par les opérateurs repose, notamment, sur l'utilisation de matériaux qui absorbent les neutrons (matériaux neutrophages) : bore, cadmium, hafnium. Ces matériaux constituent les éléments de contrôle ou de sécurité des réacteurs de recherche, éléments mobiles positionnés dans le cœur du réacteur, permettant d'ajuster et de maîtriser la réactivité de celui-ci. Leurs mouvements verticaux sont commandés par des mécanismes généralement situés au-dessus du cœur. Dans certains cas toutefois, pour faciliter l'accès aux dispositifs expérimentaux ou d'irradiation, ils peuvent être commandés par des mécanismes situés dans la partie basse du bloc-pile - cela est le cas des réacteurs d'irradiation que sont OSIRIS ou le réacteur Jules Horowitz, dans lesquels un local situé sous la piscine du réacteur abrite les mécanismes correspondants. Il en résulte qu'il convient de traiter les risques potentiels de fuite d'eau - éventuellement contaminée dans le cas d'accident qui affecterait le réacteur - vers les sous-sols des réacteurs par la mise en place de revêtements d'étanchéités dans les salles des mécanismes des absorbants.

Parmi les éléments absorbants, certains sont dédiés à la conduite du réacteur (éléments de contrôle ou pilotage), d'autres à la réalisation de l'arrêt d'urgence (éléments de sécurité). Lorsque le réacteur est arrêté, tous les absorbants sont entièrement insérés dans le réacteur qui devient alors sous-critique $(\rho<0)$, avec une marge d'antiréactivité suffisante. En état d'arrêt établi, il peut être requis, pour des raisons de sûreté, que quelques éléments de sécurité (un ou deux) soient retirés de la zone du cœur, de façon à disposer d'une antiréactivité par exemple lors d'opérations sur le cœur telles que son rechargement, en cas d'erreur de manipulation ${ }^{201}$. Lors du démarrage du réacteur, les opérateurs extraient d'abord les éléments de sécurité du cœur, tout en le maintenant sous-critique ; puis l' (ou les) élément(s) de pilotage est (sont) progressivement extrait(s) du cœur jusqu'à ce qu'il devienne critique $(\rho=0)$. Cet (ou ces) absorbant(s) est (sont) ensuite utilisé(s) pour augmenter ou diminuer la puissance du réacteur, ainsi que pour compenser l'usure du combustible au cours du fonctionnement du réacteur. Les éléments de sécurité restent quant à eux maintenus axialement hors de la zone correspondant au cœur (généralement par des électro-aimants) ; leur rôle est d'assurer, en situation incidentelle ou accidentelle, un arrêt automatique du réacteur par leur chute gravitaire ou leur insertion accélérée (par un dispositif à gaz sous pression) dans le cœur ou au droit de la zone du cœur, après la désactivation des électroaimants (figure 7.3 ).

201. Cela constitue une exigence de conception du cœur et des éléments absorbants. 


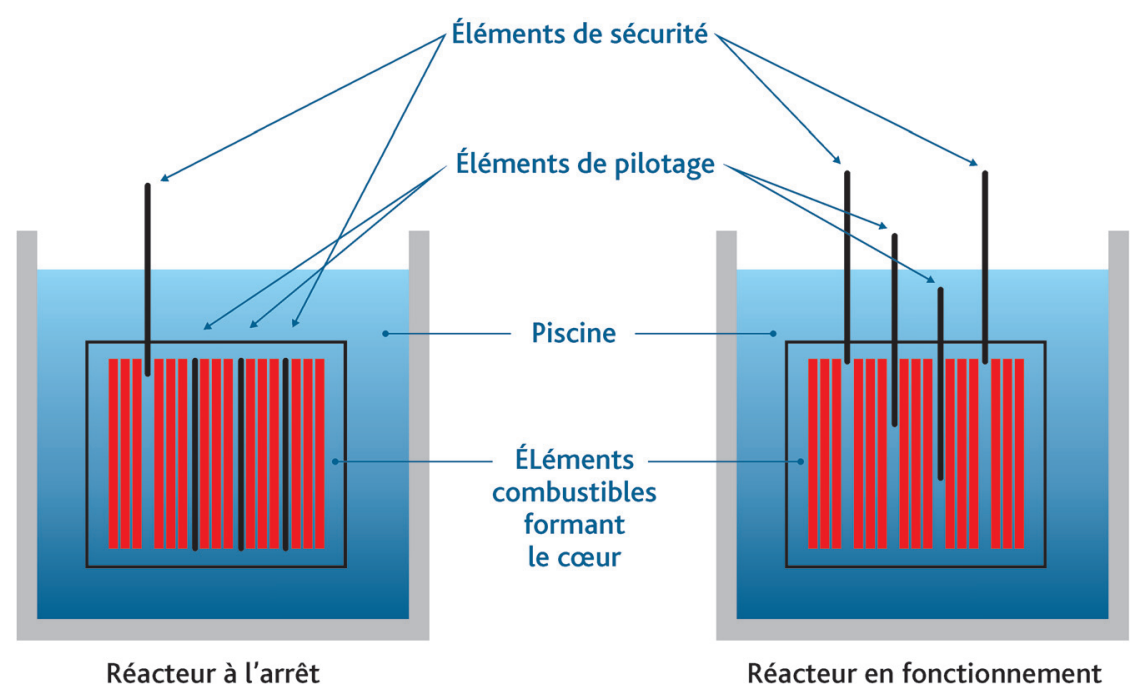

Figure 7.3. Positions des éléments de sécurité et des éléments de pilotage dans un réacteur de type piscine dans une situation d'arrêt (à gauche) et en fonctionnement (à droite). ๑ Georges Goué/IRSN.

Chaque réacteur est doté à cette fin d'un système de protection relié à des capteurs (température, pression, flux de neutrons...) redondants, dont les signaux émis sont traités selon une logique de " vote » appropriée et sûre et à des actionneurs. Les paramètres suivis et les valeurs des seuils dont le franchissement déclenche des actions de sécurité (principalement l'insertion des éléments de sécurité) sont propres à chaque réacteur ; les valeurs retenues pour ces seuils sont notamment issues des études des " conditions de fonctionnement ${ }^{202}$ ". L'insertion des éléments de sécurité peut également être déclenchée par des signaux externes au réacteur proprement dit (séisme, choc sur le bâtiment du réacteur, perte d'une dépression requise dans un local...), ainsi que par des signaux émis par des capteurs de surveillance de certains paramètres de fonctionnement de dispositifs expérimentaux, lorsque cela est jugé nécessaire pour la sûreté du réacteur.

Le système de protection d'un réacteur de recherche est un élément-clé de sa sûreté. Sa conception s'appuie généralement, autant que nécessaire, sur un certain nombre d'options visant à obtenir une fiabilité élevée : redondance, diversification technologique, séparation physique des voies de mesures, etc. L'application du critère de défaillance unique $(C D U)$ vise à ce qu'une seule défaillance ne puisse rendre un système inapte à accomplir la fonction de sûreté qui lui est attribuée. Cela peut être réalisé par la redondance, en doublant (ou plus) les éléments d'un système et en les répartissant en " voies " différentes de telle sorte que chacune d'elles puisse assurer la fonction du système. Cette redondance peut être complétée par des dispositions de séparation géographique des voies, de façon à éviter qu'une agression, par exemple un incendie, ne

202. En y ajoutant des marges de conservatisme pour tenir compte par exemple des incertitudes sur les valeurs mesurées des paramètres du réacteur, des délais d'activation des dispositifs de protection... 
puisse affecter toutes les voies en même temps (mode commun). La diversification conduit à utiliser, autant que nécessaire, des composants de conceptions ou de technologies différentes sur les voies redondantes d'un même système, pour obtenir une fiabilité appropriée de ce système. Par ailleurs, pour les composants " actifs » importants, la conception doit privilégier les " pannes sûres », c'est-à-dire qu'un composant qui tombe en panne se met dans un état favorable à la sûreté de l'installation. Par exemple, la position « sûre » des absorbants correspondant à leur insertion complète dans le cœur du réacteur, le système qui assure le déplacement de ces absorbants est conçu pour que ceux-ci chutent (pour ceux dont les mécanismes sont situés au-dessus de la zone du combustible) dans le cœur en cas de perte de leur alimentation électrique, ce qui peut être obtenu avec des électro-aimants.

Le chargement du cœur (mise en place des éléments combustibles qui constituent ce cœur) est une opération importante réalisée selon des procédures qui prévoient de multiples vérifications et contrôles. Des dispositions matérielles peuvent être mises en place pour empêcher physiquement certaines erreurs de positionnement qui pourraient dégrader la sûreté du réacteur.

Il est à noter que, dans le cadre des réexamens de sûreté périodiques, certains réacteurs de recherche ont été dotés, en complément du système de protection tel que décrit ci-avant, d'un moyen ultime permettant d'assurer l'arrêt de la réaction en chaîne à l'égard de situations accidentelles au cours desquelles une déformation du cœur pourrait empêcher ou retarder de façon rédhibitoire la chute des absorbants (par exemple en cas de fort séisme). Il peut s'agir d'un système simple d'injection d'un absorbant neutronique en solution dans l'eau du circuit primaire. À titre d'exemple, ce type de système a été mis en place pour le réacteur CABRI (injection d'eau borée) et il est retenu pour le réacteur Jules Horowitz au titre des dispositions de gestion des accidents avec fusion du cœur.

Mais, au-delà des moyens classiques de maîtrise de la réactivité décrits ci-dessus, il convient également de prévenir les défaillances susceptibles de conduire à des insertions de réactivité dans un cœur.

Ainsi, un dysfonctionnement des mécanismes des absorbants qui entraînerait un retrait voire l'éjection de ceux-ci hors de la zone du cœur - donc une insertion de réactivité -, alors que leur position requise est d'être insérée dans le cœur ou au droit de la zone du cœur afin d'en maîtriser sa réactivité, doit être étudiée, notamment dès les phases de conception d'un réacteur de recherche. Des dispositions de conception peuvent être mises en œuvre pour limiter l'amplitude ou la vitesse de retrait des absorbants, comme cela a notamment été retenu dans le cas du réacteur Jules Horowitz au cours des études de conception.

De même, des scénarios particuliers de fuites ou des ruptures de structures de blocspiles (cuves, doigts de gant...) pouvant conduire à des insertions de réactivité dans un cœur (par exemple, dans le cas du RHF, une entrée d'eau légère dans l'eau lourde qui circule dans le cœur) sont à examiner et nécessitent évidemment que des dispositions adéquates de surveillance en service de ces structures soient mises en œuvre pour les rendre suffisamment improbables. 


\subsubsection{Maîtrise du refroidissement du réacteur}

Cette fonction de sûreté ne pose pas véritablement de problème pour les réacteurs de très faible puissance, jusqu'à environ $100 \mathrm{~kW}$ en ordre de grandeur, pour lesquels la puissance thermique est dissipée naturellement dans les structures. Pour les réacteurs de puissance supérieure, le refroidissement du cœur peut être assuré - outre le caractère indispensable d'un inventaire suffisant en fluide réfrigérant - par le débit ascendant de ce fluide qui s'établit naturellement dans le cœur du réacteur (convection naturelle) mais peut également nécessiter un débit de fluide réfrigérant plus important, assuré en convection forcée. Dans ce dernier cas, le cœur du réacteur est alors refroidi par un circuit, dit circuit primaire, dont le débit est assuré par des pompes de circulation, ce circuit étant lui-même généralement refroidi par un circuit secondaire (de façon à éviter les rejets radioactifs, le fluide primaire étant susceptible d'être contaminé).

Le circuit primaire peut être complètement fermé ou être partiellement ouvert sur la piscine du réacteur. Les défaillances de ce circuit (perte de débit, baisse de pression, perte de l'inventaire en eau) sont détectées par le système de protection qui déclenche des alarmes voire la mise à l'arrêt automatique du réacteur. Il reste toutefois une puissance résiduelle à évacuer dont l'importance dépend du fonctionnement antérieur du réacteur, et notamment de la puissance de fonctionnement. Pour des réacteurs d'une puissance (en fonctionnement) de l'ordre de quelques mégawatts, le débit de convection naturelle peut être suffisant pour assurer le refroidissement du combustible du réacteur arrêté. Néanmoins, pour que ce débit s'instaure, de l'eau de la piscine dans laquelle le cœur est immergé doit pouvoir pénétrer à l'intérieur des éléments combustibles. Cette mise en communication entre le circuit primaire et l'eau de la piscine est assurée par des clapets généralement installés sur les tuyauteries d'eau de refroidissement du cœur, à l'entrée de celui-ci. Lors du fonctionnement en puissance du réacteur, ces clapets sont maintenus en position fermée par la différence de pression entre l'eau de la piscine et le circuit primaire ; ils s'ouvrent (naturellement) lorsque cette différence de pression diminue (cas d'une perte de débit primaire). Ces clapets sont en général doublés pour éviter de perdre la fonction de mise en communication de la piscine avec le circuit primaire en cas de défaillance d'un clapet (application du critère de défaillance unique). Selon la puissance résiduelle dégagée par le cœur du réacteur, il peut également être nécessaire de maintenir, dans les premières minutes qui suivent l'arrêt du réacteur, un débit de refroidissement supérieur à celui résultant de la convection naturelle. À cette fin, les pompes du circuit primaire sont équipées de volants d'inertie qui permettent d'assurer leur ralentissement progressif, maintenant ainsi une circulation de fluide primaire suffisante dans le cœur du réacteur pendant une certaine durée après l'arrêt de celui-ci. Les clapets et les volants d'inertie sont des équipements dont le fonctionnement ne nécessite pas d'alimentation en fluide (électricité, air comprimé...).

Pour certains réacteurs de recherche, la convection naturelle de l'eau dans le cœur peut même permettre d'évacuer une faible puissance de fonctionnement ; cette possibilité est prévue dans les règles générales d'exploitation de ces réacteurs. Il est à noter qu'elle permet ainsi de vérifier, en conditions normales de fonctionnement du réacteur, le bon fonctionnement des clapets et le bon établissement de la convection naturelle d'eau dans le cœur. Il 
convient toutefois de souligner que dans les réacteurs dont le cœur est refroidi en conditions normales par un débit descendant d'eau (RHF, ORPHEE), la convection naturelle ne s'instaure pas progressivement de façon immédiate, car elle nécessite une inversion du sens de l'écoulement dans le cœur ; pour de tels réacteurs, cela nécessite bien évidemment d'être vérifié lors des essais de démarrage, puis en exploitation.

Pour des réacteurs de recherche de forte puissance, comme le réacteur Jules Horowitz en cours de construction à Cadarache (d'une puissance de $100 \mathrm{MW}$ ), un débit forcé de refroidissement du cœur doit être assuré pendant plusieurs heures après l'arrêt de celui-ci, compte tenu de la puissance résiduelle dégagée. L'évacuation de cette puissance résiduelle nécessite un recours à des systèmes " actifs », comprenant des systèmes normaux et des systèmes de sauvegarde pour les situations accidentelles; ces derniers systèmes comportent des pompes et des échangeurs de chaleur avec un ou plusieurs autres circuits de refroidissement. Compte tenu de l'importance de ces circuits pour la sûreté, ils font l'objet d'exigences fortes en termes de conception, réalisation et d'exploitation (surveillance en service, maintenance...). En particulier, ces circuits sont doublés, chaque circuit étant capable à lui seul d'évacuer toute la puissance résiduelle, géographiquement séparés, leurs alimentations électriques sont secourues (batteries, groupes électrogènes) et les longueurs des tuyauteries et les nombres de brides et de raccords sont réduits pour limiter les risques de fuite ou de brèche.

Pour les réacteurs de recherche dont le cœur est placé dans une piscine, il est essentiel de maintenir l'inventaire en eau de celle-ci dans toutes les situations envisageables. En effet, le maintien de cet inventaire participe à la maîtrise de la fonction de refroidissement, et également à la protection contre les rayonnements ionisants. Une baisse du niveau d'eau dans la piscine peut résulter d'une fuite de celle-ci ou d'une fuite ou brèche d'un circuit en communication avec ladite piscine. Afin de prévenir une baisse du niveau d'eau, une disposition de conception consiste à faire en sorte que toutes les tuyauteries traversant le génie civil de la piscine débouchent au-dessus du niveau du cœur. Cela concerne notamment des circuits auxiliaires - tels que le circuit " de couche chaude » précisé plus loin au paragraphe 7.3.3, dont le rôle est de débarrasser l'eau de la piscine des impuretés déposées à sa surface, ou le circuit d'appoint d'eau qui permet de compenser l'évaporation d'eau de la piscine.

Dans le cas des réacteurs à « faisceaux sortis de neutrons », les canaux neutroniques (figure 7.4) qui traversent les parois de la piscine ne peuvent pas respecter la même disposition puisqu'ils sont situés, par définition, au niveau du cœur du réacteur ; aussi, le canal d'un faisceau de neutrons est équipé :

- généralement d'une « fenêtre ${ }^{203} »$, membrane réalisée avec un matériau suffisamment transparent aux neutrons (généralement de l'aluminium ou un alliage

203. Par exemple, les doigts de gant $\mathrm{H} 6$ et $\mathrm{H} 9$ du RHF, qui permettent l'introduction d'échantillons sur un chariot pour les soumettre au flux de neutrons le plus élevé auprès du cœur, ne disposent pas de «fenêtres ». Dans ce cas, la redondance d'étanchéité est reportée sur d’autres vannes situées en aval de la vanne de sécurité. 


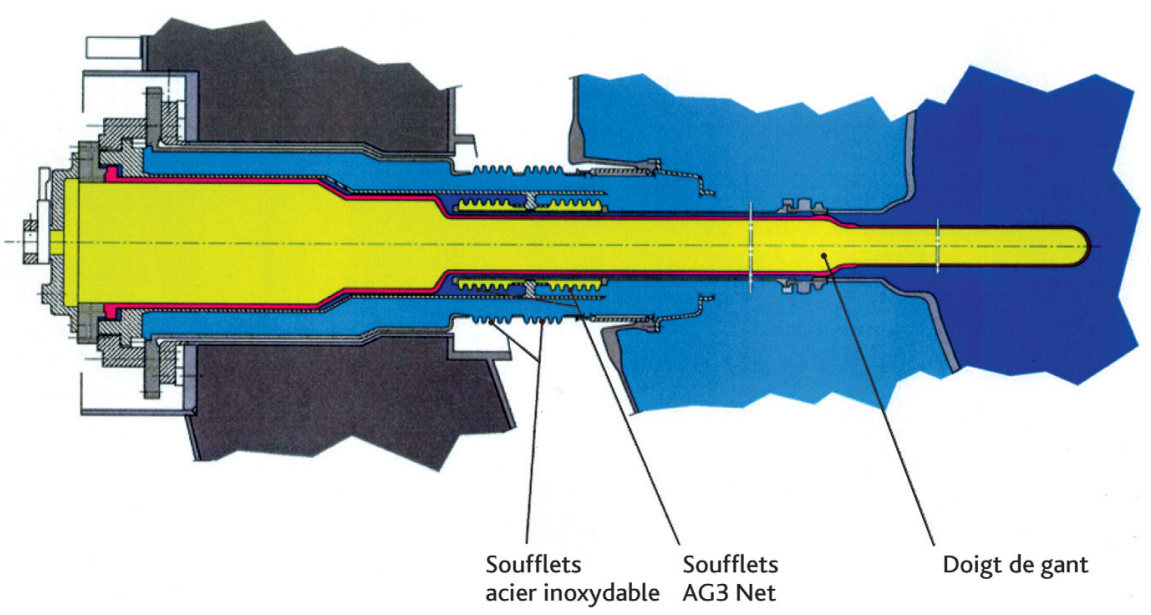

Figure 7.4. Schéma en coupe d'un doigt de gant équipant le RHF. (C) ILL.

d'aluminium), qui assure la séparation de la partie en réacteur du canal et l'air extérieur des zones dédiées aux expérimentateurs ; cette fenêtre est dimensionnée pour résister à des chargements accidentels (à une arrivée d'eau dans le canal en cas de rupture du doigt de gant, éventuellement à un accident de type BORAX dans le cœur) ;

- d'une vanne d'isolement au niveau de la paroi de la piscine, ou « vanne de sécurité », ouverte en permanence lorsque le réacteur fonctionne, permettant d'assurer une double étanchéité. En cas d'arrivée d'eau dans le doigt de gant, un automatisme peut déclencher la fermeture de cette vanne.

Par ailleurs, les canaux disposent d'obturateurs de leurs faisceaux neutroniques, organes distincts des "fenêtres » et des vannes de sécurité décrites ci-dessus.

Compte tenu de la vitesse de circulation de l'eau dans le circuit primaire, une fuite d'une portion de ce circuit en dehors de la piscine pourrait entraîner une vidange de celleci par siphonage. Pour éviter ce dénoyage, les tuyauteries du circuit primaire sont équipées de dispositifs casse-siphon (figure 7.5), dont le rôle est d'arrêter le siphonage de la piscine en injectant de l'air dans le circuit affecté par la fuite (l'envoi d'air peut être réalisé de manière passive ou par un équipement actif tel qu'une vanne).

L'un des concepts utilisé dans des réacteurs de recherche français (réacteurs de type piscine comme OSIRIS, ORPHEE et le RJH) pour réduire le risque de dénoyage du cœur du réacteur à la suite d'une brèche du circuit primaire est le concept dit "bloc-eau » (figure 7.6). Ce concept consiste à assurer une étanchéité suffisante et un volume adéquat de tous les locaux où se trouvent des portions de circuits dont une brèche pourrait conduire à une vidange de la piscine. Ainsi, en cas de rupture d'un tel circuit, l'eau s'écoule dans un local étanche jusqu'à le remplir totalement. La quantité d'eau perdue par la brèche est limitée par le volume du local, ce qui arrête la vidange de la piscine à un niveau suffisamment élevé, écartant le risque de dénoyage des éléments combustibles. 

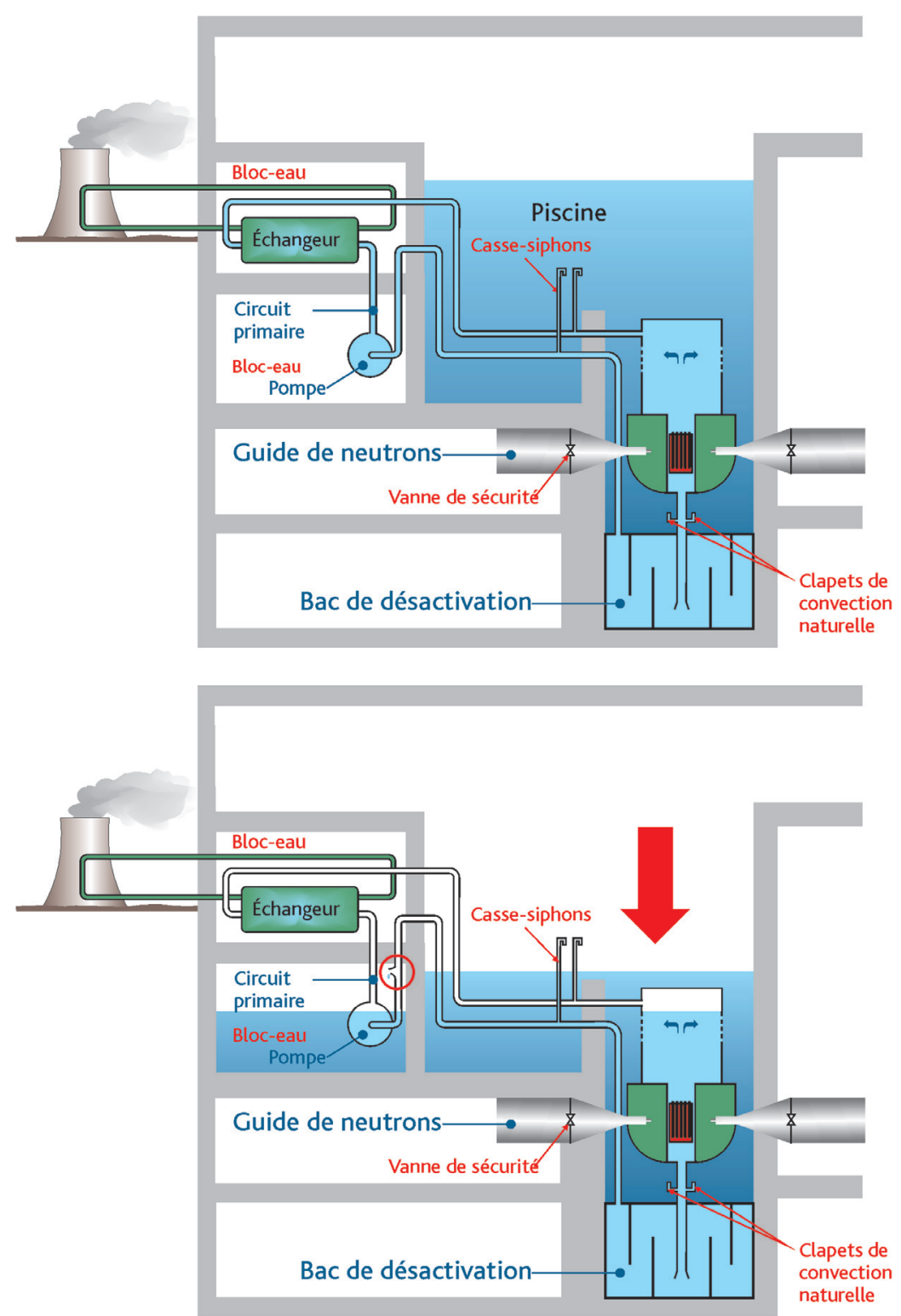

Figure 7.5. Schéma de principe de dispositifs casse-siphons « passifs » sur un circuit de refroidissement. (c) Georges Goué/IRSN.

Cela suppose toutefois que des dispositions rigoureuses d'exploitation assurent le maintien de l'étanchéité des locaux en question (maintien des portes en position fermée...), ou de conception (indication reportée en salle de commande de la position des portes...). 

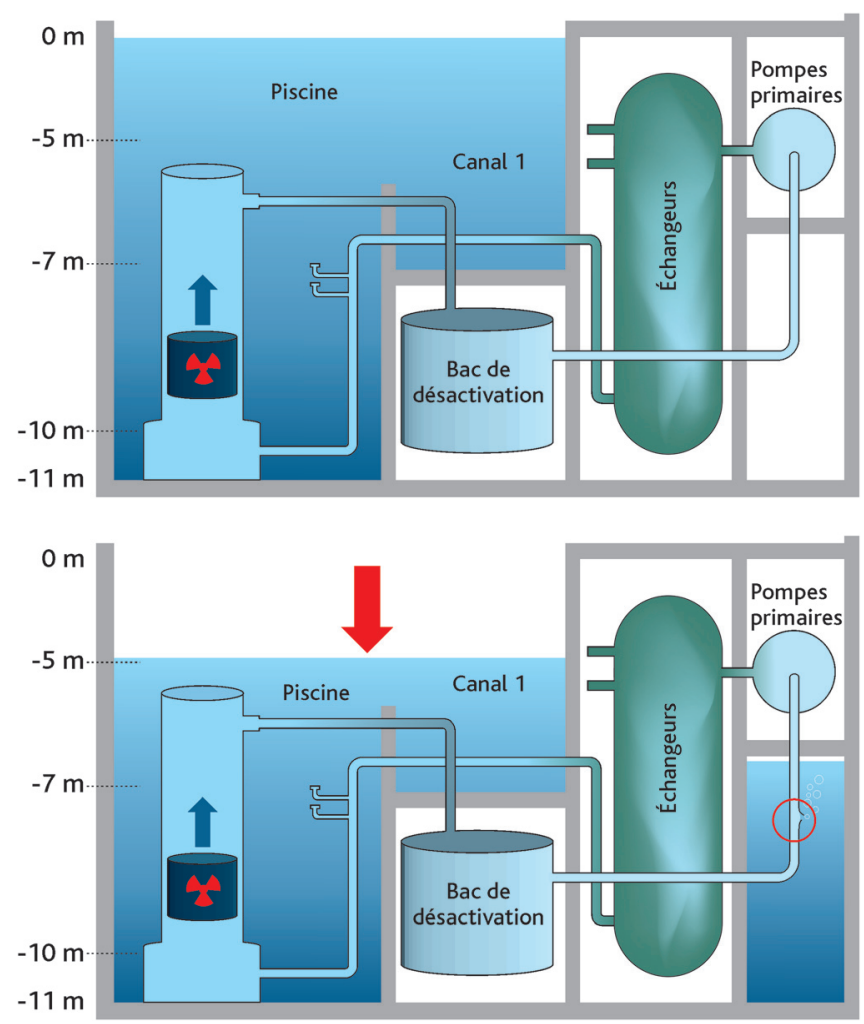

Figure 7.6. Le concept de « bloc-eau » : limitation de la baisse du niveau d'eau de la piscine en cas de brèche d'un circuit de refroidissement. @) Georges Goué/IRSN.

Enfin, il convient de rappeler que les piscines des réacteurs de recherche français utilisant du combustible à base d'alliage uranium-aluminium sont conçues pour résister à un accident de type BORAX.

\subsubsection{Maîtrise du confinement}

Le principe de la maîtrise du confinement des substances radioactives est, pour les réacteurs de recherche comme pour les autres installations nucléaires de base françaises, l'interposition de plusieurs « barrières » entre ces substances et l'environnement. En l'occurrence, à l'égard du combustible :

- la première « barrière » de confinement est constituée par les gaines métalliques des éléments combustibles ;

- une deuxième "barrière » de confinement est généralement constituée par l'enveloppe du circuit de refroidissement ; toutefois, comme cela a été indiqué précédemment, pour certains réacteurs de recherche, ce circuit communique avec la piscine et ne constitue donc pas pleinement une «barrière » de confinement ; 
- au-delà, une dernière « barrière » de confinement est constituée par le bâtiment où le cœur du réacteur est implanté (et dont la partie inférieure est constituée, selon les configurations, du radier, du plancher de la ou des piscines ou encore de locaux).

Il convient de souligner ici que cette dernière « barrière » de confinement comporte en général le bâtiment proprement dit - qui assure un confinement « passif »- et un système de ventilation et de filtration - qui assure un confinement « dynamique ».

Par ailleurs, dans les réacteurs à canaux neutroniques, les circuits d'eau lourde contiennent de l'eau tritiée formée par l'activation neutronique des atomes de deutérium de l'eau lourde. Cela impose une étanchéité appropriée de ces circuits compte tenu des risques radiologiques associés au tritium.

Le bâtiment du réacteur doit être conçu de manière à limiter les fuites vers l'environnement. À cette fin, les traversées par lesquelles passent des tuyauteries ou des câbles sont en nombre aussi limité que possible et étanchées par des matériaux appropriés. L'étanchéité d'un bâtiment n'étant jamais parfaite, le confinement passif qu'il assure est en général complété par un confinement dynamique qui maintient une légère dépression dans le bâtiment par rapport à l'extérieur (le cas particulier du RHF est abordé plus loin). Ainsi, l'air passant par les fuites du bâtiment ira préférentiellement de l'extérieur vers l'intérieur du bâtiment. Ce confinement « actif » nécessite des équipements tels que des ventilateurs d'extraction et de soufflage, complétés par des dispositifs de filtration (filtres à très haute efficacité [THE], pièges à iode [PAI]) visant à retenir les substances dangereuses avant leur rejet par l'air dans l'environnement.

En cas d'incident ou d'accident conduisant à une dispersion de substances radioactives au sein du bâtiment du réacteur, la ventilation est généralement arrêtée automatiquement lors de l'arrêt du réacteur afin d'assurer dans un premier temps un confinement statique des substances radioactives, diminuant ainsi leur dangerosité par la décroissance radioactive des produits de fission à vie courte. Dans un second temps, la ventilation peut être remise en service pour filtrer les rejets. Néanmoins, pendant la phase de confinement statique, la pression dans le bâtiment augmente (échauffement de l'air par les produits de fission en suspension) et une partie de son atmosphère peut alors être transférée sans filtration dans l'environnement par les fuites « naturelles » du bâtiment. C'est pourquoi certains réacteurs de recherche (ORPHEE, RJH) sont dotés d'une "zone de reprise des fuites " : il s'agit d'un local attenant au bâtiment du réacteur, équipé d'un système de ventilation muni de filtres, dans lequel débouchent toutes les traversées de ce bâtiment (figure 7.7) ; ce local collecte ainsi la majorité des fuites du bâtiment et les filtres avant leur rejet dans l'environnement.

Une autre disposition consiste à mettre en place autour du bâtiment une zone annulaire dans laquelle la pression est toujours maintenue à une valeur supérieure à celle qui existe à l'intérieur du bâtiment. Cette disposition a été retenue pour le RHF.

Le choix des dispositions de la dernière « barrière » de confinement est fonction de la composition et des quantités de substances radioactives susceptibles d'être relâchées en 

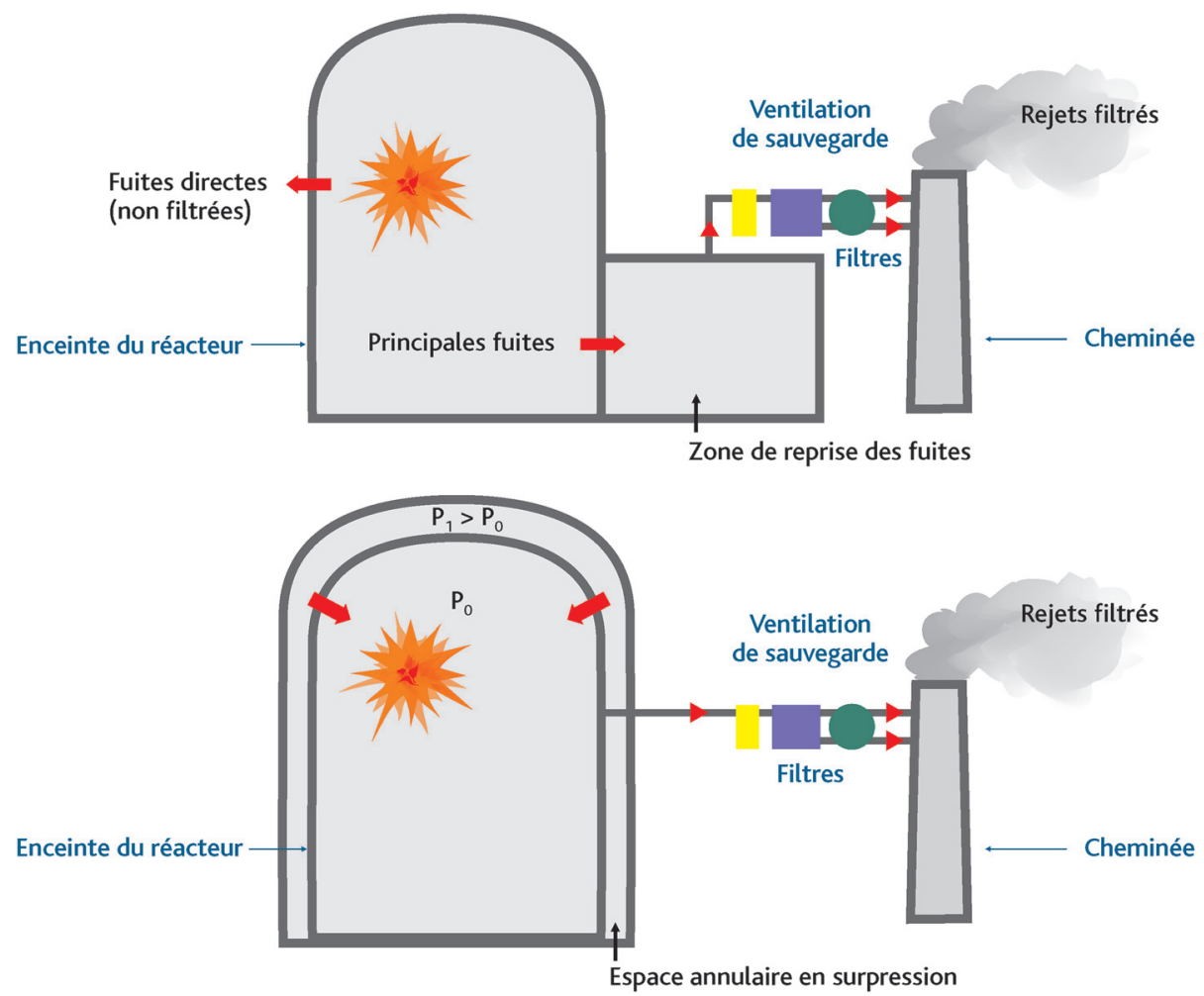

Figure 7.7. Schémas de principe de dispositions mises en place en vue d'éviter les fuites directes de l'atmosphère du bâtiment d'un réacteur vers l'environnement. (c) Georges Goué/IRSN.

conditions accidentelles et de la cinétique de ces relâchements, d'autres considérations telles que la proximité de populations pouvant également intervenir dans le choix. Pour les projets de nouveau réacteur, il s'agit d'un choix important qui doit apparaître dès le dossier d'options de sûreté, transmis à l'autorité de sûreté, et qui doit être examiné dans ce cadre.

Il convient encore de noter que la plupart des réacteurs de recherche sont équipés d'une ventilation de secours assurant une extraction d'air à un débit réduit permettant de maintenir une légère dépression dans le bâtiment du réacteur après un arrêt de la ventilation normale. Cette ventilation de secours, qui est équipée de filtres à très haute efficacité et de pièges à iode, permet d'étaler dans le temps les rejets radioactifs éventuels, de les filtrer et de mesurer la radioactivité rejetée. Le passage de la ventilation normale à la ventilation de secours est automatique en cas de dépassement d'un seuil prédéterminé d'activité dans le bâtiment du réacteur.

Deux spécificités de certains réacteurs de recherche peuvent encore être soulignées :

- comme cela a été indiqué plus haut, dans certains réacteurs de recherche, le circuit primaire communique avec la piscine. Pour protéger les opérateurs ou expérimentateurs amenés à travailler sur la margelle de la piscine, ces réacteurs disposent d'un 
système de " couche chaude » qui envoie de l'eau chaude (à $40^{\circ} \mathrm{C}$ ) à la partie supérieure de la piscine et l'aspire toujours à la partie supérieure, à l'opposé. La hauteur de la « couche chaude » est comprise entre $2 \mathrm{~m}$ et $4 \mathrm{~m}$. L'eau récupérée est épurée de façon continue sur des résines échangeuses d'ions. Grâce à la différence de température créée par la " couche chaude », les substances radioactives restent bloquées dans l'eau plus froide de la partie basse de la piscine ;

- le circuit primaire peut sortir du bâtiment du réacteur : cela est le cas du réacteur CABRI (voir la figure 5.11), pour lequel les deux réservoirs du circuit primaire situés hors du bâtiment du réacteur sont équipés d'un double fond, complété d'un bac de rétention ${ }^{204}$.

\subsubsection{Risques de criticité}

Un accident de criticité peut entraîner une irradiation grave des personnes situées à proximité de la zone concernée, pouvant entraîner leur décès. Aussi, lorsque la criticité n'est pas recherchée, que ce soit dans le cœur du réacteur lorsqu'il est à l'arrêt ou dans toute autre partie de l'installation mettant en œuvre du plutonium, de l'uranium présentant un enrichissement en isotope 235 supérieur à $1 \%$, ou certains actinides dits mineurs (curium, américium...), des dispositions sont prises pour maintenir les matières correspondantes dans un état sous-critique.

Pour déclencher une réaction en chaîne dans un milieu, une quantité suffisante de noyaux fissiles doit être mise en jeu. Il existe ainsi une masse en deçà de laquelle une réaction de fission auto-entretenue n'est physiquement pas possible pour un milieu donné. La limitation de la masse de matières fissiles ${ }^{205}$ est donc un moyen d'éviter le déclenchement d'une réaction en chaîne. Les limites associées à ce « mode de contrôle » considéré seul sont toutefois généralement faibles. Aussi, s'il est applicable à l'échelle d'un conteneur, d'une boîte à gants ou d'un laboratoire mettant en œuvre de faibles quantités de matières fissiles, il n'est pas, à lui seul, adapté à des locaux accueillant des matières fissiles en quantités importantes, comme, par exemple, les entreposages d'éléments combustibles des maquettes critiques.

Deux principes simples sont alors utilisés pour assurer la sous-criticité :

- réduire autant que possible la production de neutrons, en diminuant la probabilité des réactions de fission;

- favoriser autant que possible les fuites de neutrons hors du milieu considéré ou leur absorption par capture stérile ${ }^{206}$.

204. Il s'agit de réservoirs qui sont sous très faible pression d'eau.

205. Il est rappelé qu'un isotope est dit fissile si son noyau peut subir une fission sous l'effet d'un bombardement par des neutrons de toutes énergies (rapides ou lents). Le seul isotope fissile naturel est l'uranium 235.

206. Les neutrons sont capturés par des noyaux des métaux de structure, du modérateur, des gaines des éléments combustibles, des éléments absorbants ou encore par des noyaux fissiles mais sans donner de fission. Les neutrons sont donc perdus pour la réaction en chaîne. Les matériaux correspondants sont activés (rendus radioactifs) par ces captures. 


\section{\#FOCUS}

\section{Le ralentissement des neutrons, propice à l'entretien d'une réaction en chaîne}

Au moment de leur naissance lors d'une fission, les neutrons ont une énergie cinétique élevée (de l'ordre de $2 \mathrm{MeV}$ ) et leur probabilité de provoquer une fission est relativement faible. Mais lors de leur déplacement dans la matière, les neutrons cèdent progressivement une partie de leur énergie du fait de collisions avec les noyaux du milieu, ce qui augmente leur probabilité de capture et donc de fission. Ce processus de ralentissement des neutrons, communément appelé « modération », est d'autant plus important que les noyaux rencontrés sont légers ; l'hydrogène est donc un très bon modérateur.

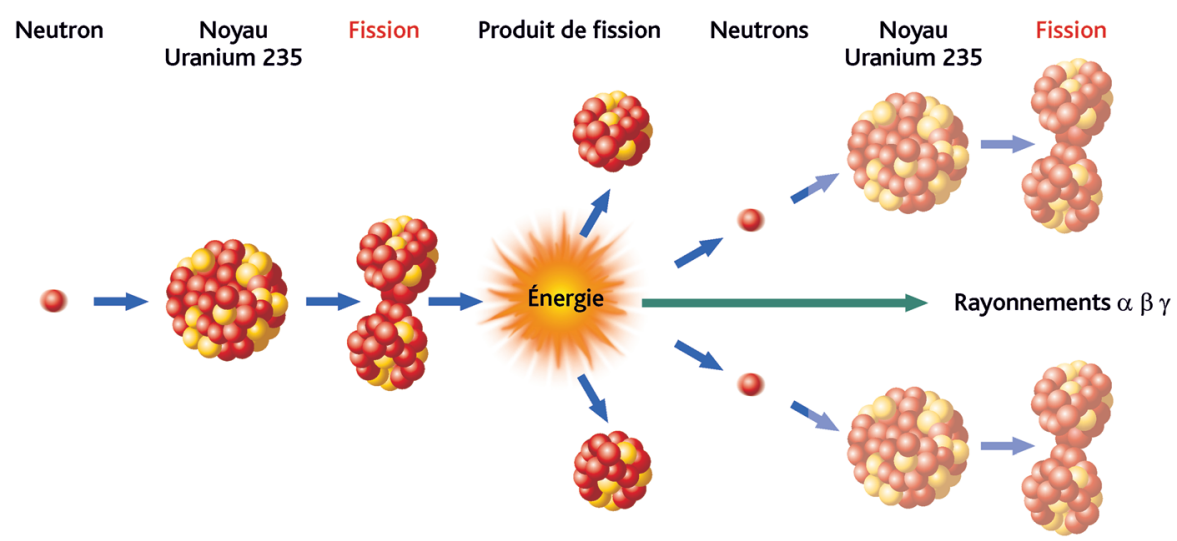

Figure 7.8. La réaction de fission de l'uranium 235. (C) Georges Goué/IRSN.

L'eau et les matériaux hydrogénés, éléments " modérateurs » (voir le focus), accroissent les probabilités de fission de l'uranium 235 et donc les risques de criticité. C'est pourquoi, dans certains locaux, l'utilisation d'eau peut être interdite, même en cas d'incendie (une poudre extinctrice spécifique, à faible teneur en hydrogène, est alors utilisée). Il s'agit d'un « mode de contrôle » par limitation de la modération.

La présence de matériaux hydrogénés dans les déchets ou autour des combustibles est à prendre en considération dans les études de criticité.

Les caractéristiques géométriques des équipements renfermant les matières fissiles ont une influence importante sur les fuites de neutrons. Le « mode de contrôle " par la géométrie offre l'avantage d'être peu sensible aux erreurs humaines en exploitation. Il 
doit cependant être retenu dès la conception des équipements chaque fois que cela est possible en tenant compte des situations et agressions pouvant conduire à des modifications de la " géométrie » des matières fissiles (corrosion, déformations accidentelles en cas d'augmentation de températures, en cas de séisme...). Des dispositions doivent par ailleurs être prises lors des modifications ou changements de ces équipements pour assurer des caractéristiques géométriques appropriées.

Les neutrons sortant d'un milieu fissile continuent leur trajectoire et peuvent être renvoyés dans leur milieu d'origine du fait des collisions avec les noyaux de matériaux (réflexion neutronique). Une fraction des neutrons sortant d'un milieu fissile peut également entrer dans un équipement proche, contenant également des matières fissiles, et y provoquer des fissions (interaction neutronique). Ces deux phénomènes doivent être pris en compte dans les études relatives aux risques de criticité.

Enfin, la maîtrise des risques de criticité peut être obtenue en ayant recours à l'emploi de "poisons neutroniques ", tels que le bore, le cadmium ou le hafnium, qui sont particulièrement efficaces pour absorber les neutrons.

Le choix d'un (ou plusieurs) « mode(s) de contrôle » pour une installation (piscine d'entreposage d'éléments combustibles, magasin d'entreposage de plaquettes ou réglettes pour la maquette MASURCA...) peut conduire à définir des limites strictes pour certains paramètres bien identifiés qui tiennent compte non seulement des conditions normales dans laquelle se trouve les matières fissiles mais aussi des situations perturbées envisageables (séisme...). Les matières susceptibles d'être présentes dans l'environnement (proche) des matières nucléaires étudiées sont évidemment à prendre en compte dans les études de criticité, car elles peuvent interagir neutroniquement avec elles et accroître les risques de criticité.

Dans un réacteur de recherche, les éléments combustibles étant fréquemment manipulés par les opérateurs, la prévention des risques de criticité peut reposer en grande partie, dans certaines phases de leur manipulation, sur des dispositions organisationnelles et humaines visant au respect des limitations définies au terme des études. Ces dispositions peuvent comporter des points d'arrêt dans les manipulations, l'utilisation de gabarits permettant de ne pas dépasser un nombre admissible de réglettes ou plaquettes de combustible comme dans le cas de MASURCA. De telles dispositions ont été adoptées à l'issue du réexamen de sûreté effectué en 2005-2006, sur la base d'une analyse des risques de criticité tenant compte des facteurs organisationnels et humains pour cette installation.

\subsection{Prise en compte des agressions}

Comme pour les autres installations nucléaires de base, les agressions à considérer relèvent de deux types :

- les agressions internes, dont l'origine est à l'intérieur de l'installation,

- les agressions externes, dont l'origine est à l'extérieur de l'installation. 


\subsubsection{Agressions internes}

Les principales agressions internes pouvant affecter la sûreté d'un réacteur de recherche sont les inondations (à la suite d'une fuite ou de la rupture d'une tuyauterie ou d'un réservoir), les incendies et les explosions, ainsi que les chutes de charge. Les réacteurs de recherche n'étant généralement pas refroidis avec des fluides à haute pression, les risques de « fouettement ${ }^{207}$ » de tuyauteries sont moindres que dans le cas des réacteurs à eau sous pression par exemple ; ils peuvent toutefois devoir être traités pour des boucles expérimentales. Pour chaque type d'agression, les conséquences sur la sûreté du réacteur doivent être évaluées et des dispositions appropriées être mises en œuvre en termes de prévention et de limitation des conséquences. Les fonctions de sûreté ne doivent pas être rendues indisponibles.

Concernant les inondations internes, les équipements électriques constituent des équipements sensibles, leur contact avec de l'eau pouvant entraîner des courts-circuits et des départs de feu.

Les locaux qui contiennent (ou peuvent contenir) des matières fissiles sont également sensibles en cas d'inondation, compte tenu du risque de criticité.

Des dispositions telles que des murets à l'entrée des locaux et la surélévation des armoires d'entreposage de matières fissiles peuvent être retenues. En outre, il convient d'éviter, autant que faire se peut, que des tuyauteries d'eau cheminent dans les locaux sensibles ou abritent des équipements sensibles en cas d'inondation.

Concernant les inondations externes, un revêtement d'étanchéité peut être mis en place dans les zones appropriées d'une installation en vue de prévenir une inondation à l'intérieur de celle-ci par de l'eau qui viendrait de l'extérieur du bâtiment de cette installation (au niveau du sol ou par remontée de nappe phréatique).

Par ailleurs, les multiples équipements (électriques) associés aux expériences peuvent compliquer la maîtrise des risques d'incendie dans un réacteur de recherche, qui doit être assurée tout au long de son exploitation. Un incendie est survenu au mois d'août 1979 dans le local abritant les armoires d'électronique de l'hodoscope ${ }^{208}$ du réacteur CABRI. L'origine du feu a été attribuée à l'échauffement d'un transformateur situé en partie basse d'une armoire. Cette armoire était fermée sur toutes ses faces, de sorte que l'incendie était déjà significatif lorsque le détecteur a donné l'alarme. Une fumée opaque et corrosive s'est répandue rapidement dans plusieurs salles, ce qui gêna considérablement les investigations et la lutte contre l'incendie, le foyer le plus actif étant masqué par l'armoire et les salles ne disposant pas alors d'un système de désenfumage. Il s'ensuivit, pendant un temps assez long, une erreur d'appréciation quant à la salle où le sinistre

207. La rupture complète d'une tuyauterie peut conduire à des mouvements des deux tronçons opposés, ce qui est appelé fouettement, plus ou moins violents selon la pression du fluide à l'intérieur du circuit concerné.

208. Il s'agit d'un dispositif permettant d'observer le comportement d'éléments combustibles placés dans la section d'essai du réacteur CABRI lors des expérimentations simulant des conditions accidentelles menant à la fusion et à la coulée de combustible, plus précisément les mouvements de la matière fissile. 
s'était déclaré. Le feu fut finalement combattu à l'eau diffusée et ne fut totalement maîtrisé qu'au bout de deux heures. Toutes les armoires d'électronique ${ }^{209}$ de l'hodoscope furent détruites et ce dernier fut inutilisable pendant huit mois.

Une règle fondamentale de sûreté a été établie en 1991 spécifiquement pour les réacteurs de recherche en matière de risques d'incendie (voir le paragraphe 6.2). Plus récemment, en 2014 , une décision de l'ASN ${ }^{210}$ a fixé les règles applicables aux installations nucléaires de base en général pour la maîtrise de ce risque. Le principe général de défense en profondeur y est ici décliné en quatre niveaux :

- «la prévention des départs de feu;

- la détection et l'extinction rapide des départs de feu pour, d'une part, empêcher que ceux-ci ne conduisent à un incendie et d'autre part, rétablir une situation de fonctionnement normal ou, à défaut, atteindre puis maintenir un état sûr ${ }^{211}$ de l'INB ;

- la limitation de l'aggravation et de la propagation d'un incendie qui n'aurait pas pu être maîtrisé afin de minimiser son impact sur la sûreté nucléaire, et de permettre l'atteinte ou le maintien d'un état sûr de l'INB ;

- la gestion des situations d'accident résultant d'un incendie n'ayant pu être maîtrisé de façon à limiter les conséquences pour les personnes et l'environnement. "

Les trois premiers niveaux visent à ne pas compromettre les fonctions fondamentales de sûreté ; les équipements qui assurent ces fonctions doivent bien évidemment être protégés des effets d'un incendie.

Comme pour les autres installations nucléaires de base, les départs de feu sont prévenus dans les réacteurs de recherche par l'utilisation autant que possible de matériaux peu inflammables et par la limitation des matières combustibles au strict nécessaire dans les locaux ou les zones qui leur sont affectés (piscines d'entreposage, magasins...).

Par ailleurs, ils sont équipés de systèmes de détection d'incendie qui informent les opérateurs en cas de départ de feu et qui peuvent éventuellement déclencher des actions automatiques, comme l'arrêt du soufflage d'air du système de ventilation, la fermeture de clapets coupe-feu pour limiter la propagation de l'incendie.

Enfin, des moyens de lutte adaptés aux types d'incendie envisagés et aux équipements à protéger équipent les réacteurs de recherche. Certaines spécificités peuvent toutefois être soulignées : par exemple, dans les locaux d'entreposage et de manutention des éléments constitutifs des cœurs (plaquettes et réglettes de combustible, de sodium

209. Certains documents font en outre état d'une détérioration de relais du système de protection du réacteur par les fumées qui se sont propagées.

210. Décision ASN n²014-DC-0417 du 28 janvier 2014.

211. Le guide $A S N n^{\circ} 22$ relatif à la conception des réacteurs à eau sous pression, diffusé en 2017, définit ainsi un état sûr : « état stabilisé d'une INB dans lequel la sous-criticité l'évacuation de la puissance résiduelle et le confinement des substances radioactives sont assurés durablement. » 
solide...) de l'installation MASURCA, des dispositifs d'extinction utilisant des poudres à base de graphite sont installés pour les cas de feu pouvant impliquer des éléments de sodium solide, compte tenu du caractère énergétique d'une réaction sodium-eau (feu de sodium, émission d'aérosols d'oxyde et de peroxyde de sodium...). Par ailleurs, comme cela a déjà été indiqué au paragraphe 7.3.4, l'utilisation d'eau peut être interdite dans certains locaux à risque de criticité, l'eau étant un modérateur neutronique : tel est le cas pour les zones d'entreposage et de manutention des éléments fissiles dans le bâtiment de stockage et manutention (BSM) de MASURCA.

Afin de limiter la propagation d'un incendie qui n'aurait pas été éteint rapidement, les installations sont découpées en secteurs de feu. Un secteur de feu est un volume délimité par des parois telles qu'un incendie survenant à l'intérieur ne puisse pas se propager à l'extérieur ou inversement pendant une durée définie, permettant la mise en œuvre de moyens d'extinction. La mise en place de secteurs de feu permet d'éviter de considérer la perte d'une fonction de sûreté par mode commun dès lors que la fonction est assurée par deux systèmes redondants, implantés dans deux secteurs de feu distincts. C'est généralement le cas pour le système de protection d'un réacteur nucléaire (qui déclenche l'arrêt d'urgence), pour les alimentations électriques, etc.

Pour les réacteurs de recherche anciens, la mise en place de dispositions de sectorisation n'a toutefois pas été prévue dès leur conception; ce point fait l'objet d'une attention particulière, notamment lors des réexamens de sûreté à l'occasion desquels la possibilité de réaliser des améliorations à ce sujet est tout particulièrement étudiée.

Il convient de mentionner la découverte de certains écarts de conformité relatives à des dispositions de sectorisation dans les installations nucléaires de base en général, par l'exploitant lui-même ou lors de visites de surveillance de l'Autorité de sûreté nucléaire. Dans le cadre de l'approfondissement de la réduction des risques associés aux incendies, des études en cours concernent les possibilités de propagation, hors d'un secteur siège d'un incendie, de gaz imbrûlés et de suies, qui peuvent mettre en cause des voies redondantes de systèmes classés de sûreté implantées dans d'autres secteurs de feu. L'IRSN mène des travaux de recherche et de développement sur ce sujet ${ }^{212}$.

Pour les réacteurs de recherche, la prévention des risques associés d'explosion repose, d'une manière générale, sur la réduction de leurs sources possibles et leur éloignement des équipements importants pour la sûreté. Les risques d'explosion liés aux dispositifs expérimentaux mis en place dans les réacteurs de recherche (y compris les équipements associés « à demeure » comme les « sources froides » et les « sources chaudes ») sont abordés plus loin.

Concernant les chutes de charge pouvant survenir lors des manutentions d'objets relativement lourds (assemblages ou éléments combustibles, dispositifs expérimentaux, emballages de transport...), la prévention repose sur l'adoption de dispositions robustes

212. Voir l'ouvrage « État des recherches dans le domaine de la sûreté des réacteurs à eau sous pression », Collection sciences et techniques, IRSN/EDP Sciences, 2017. 
de conception et de surveillance en service des engins de levage. Néanmoins, il n'est généralement pas possible d'exclure toute chute de telles charges. C'est pourquoi, dans un certain nombre de piscines, ont été installées des plaques métalliques placées sur des absorbeurs de chocs (parfois appelés « plots résilients »), en vue de protéger le fond - ou certaines zones du fond - de ces piscines à l'égard de chutes d'objets. De même, certains réacteurs de recherche sont dotés de dispositifs dits " d'accompagnement de charge » qui permettraient de ralentir la chute d'une charge en cours de manutention en cas de défaillance du moyen de levage.

\subsubsection{Agressions externes}

Les agressions externes peuvent être d'origine humaine ${ }^{213}$ ou naturelle. Les agressions envisageables d'origine humaine résultent de la présence d'industries et de voies de communication dans l'environnement proche de l'installation considérée. Les agressions naturelles envisageables dépendent étroitement du site où est construit le réacteur de recherche. Parmi les différentes agressions externes envisageables d'origine naturelle, on peut citer les séismes, les inondations, les incendies, la foudre, les vents violents et les tornades, ainsi que les températures extrêmes.

Pour un nouveau projet de réacteur de recherche, la prévention des agressions externes relève d'abord du choix du site, de façon à éviter que ce choix ne conduise à des difficultés rédhibitoires quant à la protection et au dimensionnement des installations. En tout état de cause, il convient de déterminer les « aléas » à retenir pour l'installation et la méthode de détermination est différente suivant le type d'agression.

Il est à noter que, de façon générale, un certain nombre de réacteurs de recherche ont été construits au voisinage ou dans le périmètre de centres d'études ou d'universités (comme ce fut le cas du réacteur universitaire de Strasbourg, arrêté depuis).

Pour les agressions envisageables dues à l'environnement industriel (usines de procédés chimiques, etc.) ou à des activités humaines (transports de toutes natures, y compris les transports aériens - civils et militaires), la détermination des aléas à retenir est en général effectuée sur la base d'une analyse de type probabiliste. Si la probabilité de l'agression est très faible, de l'ordre de $10^{-7}$ par an, la pratique actuelle retenue dans les règles fondamentales de sûreté (voir le chapitre 6) retient que l'agression est suffisamment improbable pour qu'elle puisse ne pas être considérée pour la protection et le dimensionnement, ou la vérification du dimensionnement de l'installation considérée.

Les principaux risques associés aux voies de communication terrestre et à l'environnement industriel d'un réacteur de recherche sont l'explosion d'un nuage de gaz (explosion d'un camion transportant des matières dangereuses ou d'un entrepôt contenant de telles matières) ou la dérive d'un nuage de gaz toxique jusqu'à l'installation. Ces risques ont été particulièrement étudiés pour le RHF, implanté dans l'agglomération grenobloise et à proximité d'une zone industrielle, d'une autoroute et d'une gare de

213. Les actes de malveillance ne sont pas traités dans le présent ouvrage. 
triage. Les dispositions prises à la conception sont complétées, en tant que de besoin lors des réexamens de sûreté, en considérant de surcroît la préparation à d'éventuelles situations d'urgence (gestion de crise).

La caractérisation des agressions d'origine naturelle à retenir est établie sur la base des données historiques disponibles, extrapolées pour définir une agression majorante, éventuellement associée à une période de retour donnée.

La détermination des aléas relatifs aux différentes causes possibles d'inondations externes et la définition des moyens de protection adaptés pour y faire face fait l'objet du guide $n^{\circ} 13$ de l'ASN, diffusé en $2013^{214}$. La prise en compte du risque d'inondation nécessite d'explorer pour chaque site d'un réacteur de recherche toutes les causes possibles: fortes pluies, crue de rivière d'origine pluviale, crue en cas de rupture de barrage(s), remontée de nappe phréatique, etc. Les réacteurs de recherche français ne sont pas implantés en bords de mer, ce qui permet d'écarter les risques de fortes marées, de tsunamis...

À l'égard des risques de fortes pluies, les systèmes d'évacuation des eaux pluviales sont dimensionnés de telle sorte qu'il n'y ait pas d'entrée d'eau dans l'installation et si besoin, des surbots sont installés au niveau de certaines portes.

La question de la sûreté du RHF en cas d'une rupture de barrage a été plusieurs fois réexaminée dans le cadre des réexamens périodiques, compte tenu de l'implantation de cette installation au confluent du Drac et de l'Isère - rivières coupées par de nombreux barrages -, ainsi que dans le cadre des évaluations complémentaires de sûreté menées en France après l'accident de la centrale nucléaire de Fukushima Daiichi ; ce sujet sera évoqué au paragraphe 10.2 .

La façon de traiter les risques sismiques est davantage développée ci-après. Le risque de survenue d'un séisme d'intensité notable peut être relativement élevé pour certains réacteurs de recherche compte tenu de leur implantation respective. Il convient en outre de souligner qu'une telle agression sollicite simultanément l'ensemble d'une installation et peut conduire à des défaillances de modes communs.

À l'égard des risques sismiques, jusque dans les années 1970, les réacteurs de recherche construits en France ont été conçus selon les règles parasismiques en vigueur $^{215}$, qui ne concernaient pas spécifiquement les ouvrages particuliers comme les installations nucléaires de base. En 1974, à l'occasion du développement des réacteurs de puissance en France, le Département de sûreté nucléaire (DSN) du CEA ${ }^{216}$ établit alors (pour le groupe permanent en charge des réacteurs nucléaires) un rapport, dénommé rapport DSN 50 et intitulé «Protection des centrales vis-à-vis des séismes ». Ce rapport recensait les pratiques française et internationale en la matière et formulait un certain

214. Voir à ce sujet le document « L'aléa inondation - État de l'art préalable à l'élaboration du guide inondation pour les installations nucléaires », rapport IRSN, 2013.

215. Recommandations AS 55 (de 1955), établies à la suite du séisme d'Orléansville en Algérie en 1954, règles parasismiques $\mathrm{PS} 62,64,67,69 \ldots$

216. L'IPSN n'a été créé au sein du CEA qu'en 1976. 
nombre de propositions, concernant notamment la détermination des spectres ${ }^{217}$ de sol à utiliser (dans le cadre de l'utilisation d'une méthode dynamique analytique) pour l'évaluation de la « réponse » de structures à un séisme. Ces éléments constituèrent une base de travail pour les exploitants et préfigurèrent la règle fondamentale de sûreté RFS I.2.c, diffusée en 1981 par le Service central de sûreté des installations nucléaires, applicable aux réacteurs à eau sous pression et proposant une méthode déterministe acceptable pour la détermination des mouvements sismiques à prendre en compte pour la conception des réacteurs précités à l'égard des risques sismiques. En 1992, la règle RFS I.1.C a élargi l'application de cette méthode à l'ensemble des installations nucléaires de base, dont les réacteurs de recherche. L'application de la méthode commence par le recensement, à partir des données géologiques et sismologiques disponibles, des séismes les plus forts connus (sur une période d'environ 1000 ans) dans l'environnement du site de l'installation afin de déterminer l'intensité d'un séisme dit séisme maximal historiquement vraisemblable (SMHV). Une majoration (d'une unité) de l'intensité conduit au séisme dit séisme majoré de sécurité (SMS) à retenir comme référence pour la sûreté de l'installation considérée. En 2001, une nouvelle règle fondamentale de sûreté, la RFS n²001-01, préparée avec les experts de l'IPSN, a été diffusée par l'autorité de sûreté, après plusieurs années de discussions entre les parties concernées. Cette nouvelle RFS conserve une approche déterministe mais introduit, notamment, quelques développements complémentaires concernant la prise en compte des paléoséismes et des effets de site. De plus, pour les sites à très faible risque sismique, un spectre minimal forfaitaire est prévu, pour lequel l'accélération maximale du sol (accélération correspondant à une fréquence infinie, aussi appelée Peak Ground Acceleration [PGA]) est calée à 0,1 fois l'accélération terrestre $(\mathrm{g})^{218}$.

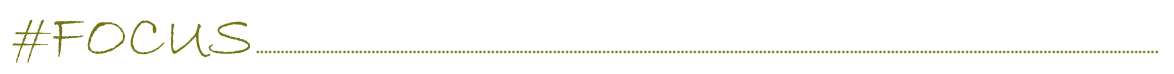

\section{Paléoséismes - Effets de site}

Les paléoséismes sont d'anciens forts séismes, identifiables par les traces qu'ils ont laissées dans les dépôts géologiques du quaternaire. Leur étude a pour objectifs d'apprécier la survenue de tels séismes, de les caractériser et de compléter la connaissance de la sismicité d'un site au-delà de la période instrumentale (dernier siècle) et de la période historique (approximativement le dernier millénaire en France).

Les effets de site à étudier correspondent aux amplifications possibles des mouvements sismiques, dues à la présence d'une couche de sol de faible résistance mécanique à proximité de la surface (30 mètres sous la surface).

217. Réponse, en termes d'accélérations, de résonateurs de différentes fréquences propres, soumis au séisme considéré.

218. Pour les zones moyennement sismiques, les accélérations peuvent atteindre environ $0,3 \mathrm{~g}$. Pour les zones très sismiques (Japon, Turquie...), les accélérations peuvent atteindre des valeurs comprises entre 0,4 et $0,6 \mathrm{~g}$. 
En outre, dans certains cas particuliers, la géométrie complexe ou la présence d'une forte épaisseur des couches sédimentaires (par exemple, d'une cuvette sédimentaire) peut conduire à une amplification ou à une augmentation de la durée du mouvement sismique. Ces effets, dits effets de site particuliers, ne sont pas dus uniquement aux propriétés superficielles du sol dans les 30 mètres sous sa surface.

Les effets de site sont particulièrement à prendre en compte pour un réacteur de recherche tel que le RHF à Grenoble, du fait de la présence d'une cuvette alluvionnaire (schéma en bas de la figure 7.9). Les installations du centre de Saclay sont sur un sol de type alluvionnaire (mais dans une zone faiblement sismique), tandis que celles du centre de Cadarache sont sur un sol mêlant roches et sédiments.
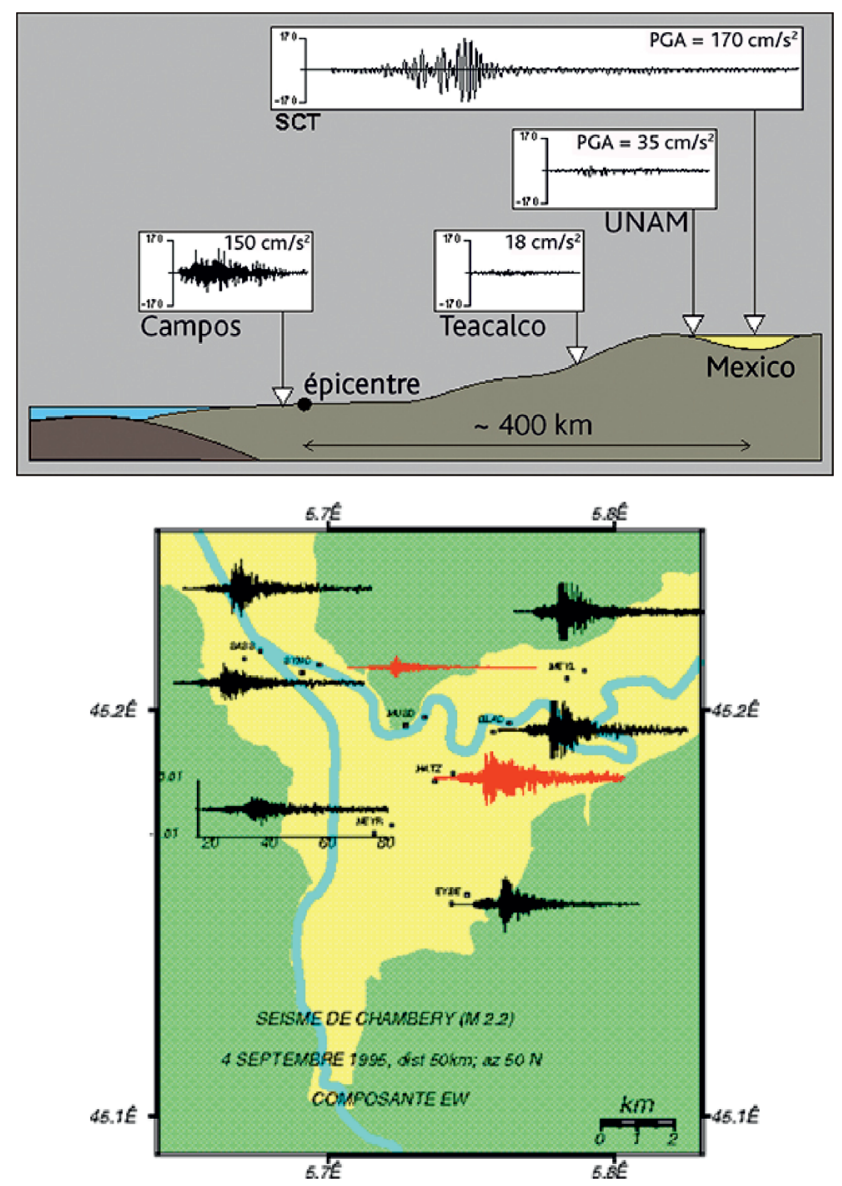

Figure 7.9. En haut, exemple d'effets de site dans la zone lacustre de Mexico ( J.F. Semblat, A. Pecker (IUSS Press, 2009) ; en bas, exemple d'effets de site du bassin de Grenoble lors du séisme de Chambery en 1996. C DR. 
Tous les composants d'une installation subissant (simultanément) les effets des mouvements sismiques, la sûreté de celle-ci repose sur une conception (y compris le dimensionnement) robuste ${ }^{219} d^{\prime}$ un ensemble d'équipements sélectionnés qui permettent d'assurer les fonctions fondamentales de sûreté et si nécessaire, dans le cas d'un réacteur nucléaire, sa mise à l'état sûr, ou, dans le cadre d'une approche " séisme-événement » (voir le paragraphe 7.1.2), pour éviter qu'ils ne puissent (en cas de chute par exemple) venir agresser des équipements importants pour la sûreté.

La vérification du bon comportement des équipements sous sollicitations sismiques peut être réalisée par le calcul (c'est le cas pour le génie civil ou les structures métalliques). Elle peut l'être aussi par des essais en vraie grandeur sur table vibrante pour des équipements tels que les clapets de convection naturelle, des groupes électrogènes ou les armoires électriques, ou encore les absorbants de sécurité pour vérifier leur aptitude à s'insérer correctement dans la zone du cœur en cas de séisme, y compris en cas de déformation de ce dernier sous l'effet d'un séisme.

Parmi les équipements pouvant venir agresser des équipements importants pour la sûreté ${ }^{220}$, les ponts de manutention font l'objet d'une attention particulière - et notamment pour les réacteurs de recherche dont le cœur est directement accessible par la piscine (circuit primaire ouvert). La chute d'un pont de manutention ou de son chariot pourrait en effet occasionner des dégâts importants sur la partie supérieure d'un cœur, l'instrumentation, les mécanismes de commande des absorbants, une boucle sous pression, une tuyauterie de refroidissement du réacteur, des clapets de convection naturelle, des éléments combustibles entreposés..., entraînant par exemple un accident de criticité ou de réactivité, un rejet brutal de vapeur, ou empêchant l'insertion d'absorbants de sécurité dans le cœur, le refroidissement correct de celui-ci... La présence, sur de nombreux réacteurs de recherche français, d'un dispositif déclenchant l'arrêt d'urgence lors de sollicitations sismiques dépassant un certain niveau permet de limiter les conséquences de la chute d'un pont ou de son chariot occasionnée par un séisme : la sous-criticité peut en effet être alors assurée - sans pour autant que le bon refroidissement des éléments combustibles puisse être nécessairement garanti, du fait des déformations de ces éléments. La prévention de la chute d'un tel pont ou de son chariot, toutes causes prises en compte, apparaît donc essentielle : conception, dimensionnement et réalisation suivant les meilleures pratiques éprouvées, mise en œuvre de contrôles en service (dont les contrôles de la règlementation sur les appareils et accessoires de levage), règles d'exploitation réduisant au strict nécessaire les survols, par le pont, de zones présentant des risques...

219. Cela fait l'objet notamment du guide de l'ASN 2/01 du 26 mai 2006, relatif à la prise en compte du risque sismique pour la conception (parasismique) des ouvrages de génie civil d'INB, à l'exception des installations de stockage à long terme des déchets radioactifs.

220. Il est à noter que certains ponts peuvent être traités comme des équipements importants pour la sûreté et faire l'objet des dispositions générales adoptées à l'égard de tels équipements en matière par exemple d'inspection en service, de maintenance, de traçabilité des modifications... 
\#FOCUS

\section{Amélioration des connaissances sur le comportement de structures soumises à des sollicitations sismiques : le cas des ponts de manutention}

L'évaluation du comportement d'un pont avec son chariot soumis à des sollicitations sismiques n'est pas aisée (glissements possibles, chocs sur des butées...). Des difficultés sont apparues à ce sujet à l'occasion de certains réexamens de sûreté, en particulier lorsque la réévaluation des mouvements sismiques a conduit à une augmentation significative des sollicitations. Dans les années 2000, l'IRSN a engagé, en collaboration avec le CEA, des études sur ce sujet, comprenant des essais réalisés sur la table vibrante AZALÉE du centre d'études CEA de Saclay (figure 7.10). Ces travaux visent à mieux comprendre la « réponse » de telles structures complexes à des mouvements sismiques - et à apprécier la robustesse des différentes méthodes simplifiées qui ont pu être utilisées par les exploitants pour l'étude de telles structures.

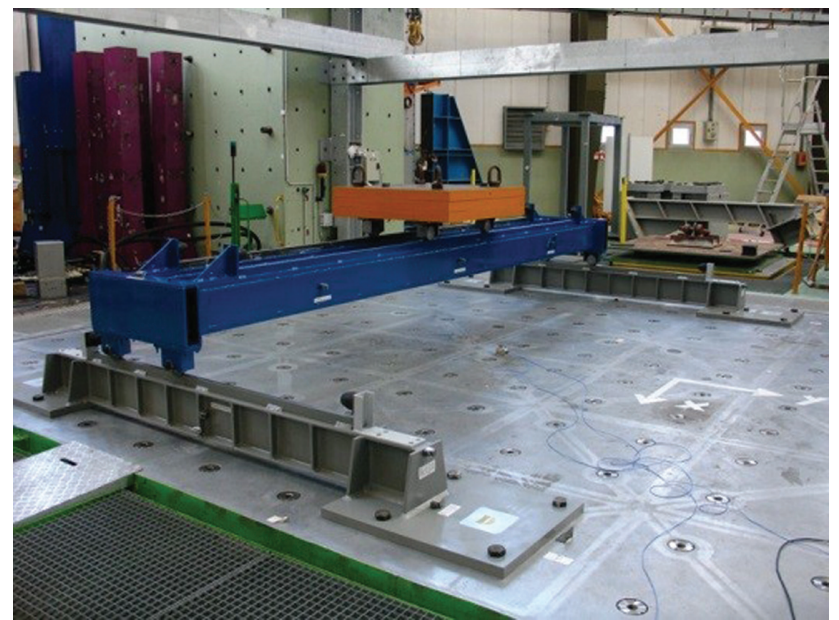

Figure 7.10. Pont de manutention testé sur la table vibrante AZALÉE du centre d'études CEA de Saclay. (C) CEA.

Il est par ailleurs à noter que certains dispositifs permettent de limiter les mouvements transmis à un bâtiment et à ses équipements en cas de séisme. Ainsi, dans le cas du réacteur Jules Horowitz $(\mathrm{RJH})$ implanté sur le site de Cadarache, dans une région marquée par de forts séismes historiques - séisme de Lambesc en 1909 -, le radier du bâtiment du réacteur a été placé sur des plots en béton armé sur lesquels sont installées des couches de matériau élastomère et d'acier (figure 7.11). Ce dispositif (dit « d'isolation parasismique ») réduit les sollicitations sismiques horizontales dans 


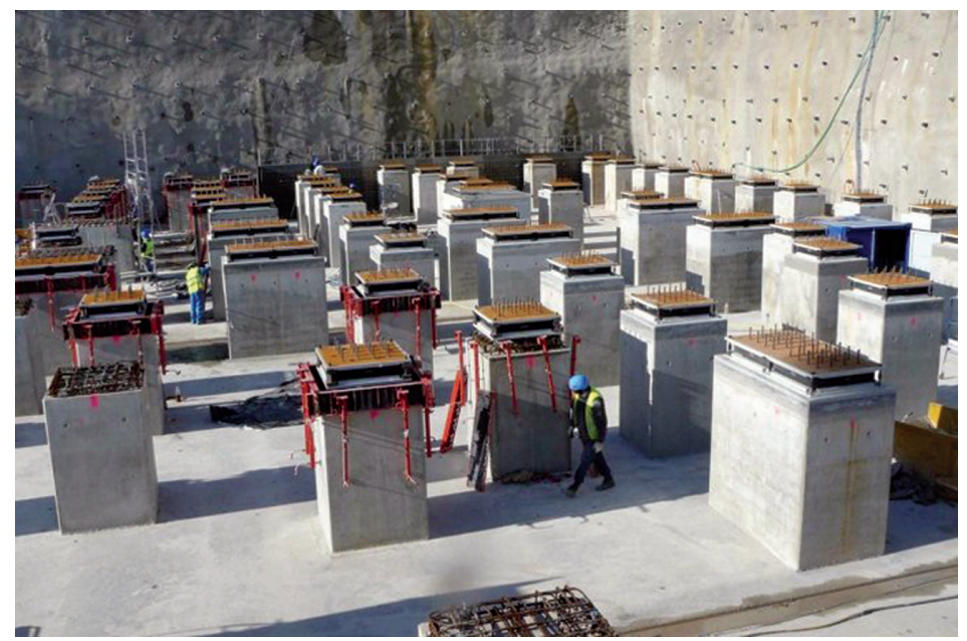

Figure 7.11. Vue, lors de la construction du réacteur Jules Horowitz (2009), des plots avec leurs patins antisismiques, d'après Le Blog des Énergies. (๑) DR.

les hautes fréquences ${ }^{221}$ pour les équipements installés sur les planchers dans ce bâtiment, pour les ponts de manutention... Bien entendu, la question se pose du maintien en l'état des plots et des couches de matériau élastomère tout au long de l'exploitation du réacteur, de leur vieillissement. C'est pourquoi il est nécessaire que ces plots et les couches de matériau élastomère soient remplaçables si, au cours des visites prévues, des anomalies devaient être constatées.

Il est également à noter, dans le cas du RJH, l'adoption d'une disposition de conception de nature à réduire les risques de chocs par mouvements différentiels entre le bâtiment du réacteur et le bâtiment des annexes : ces deux bâtiments (« unité nucléaire ») sont en effet implantés sur un seul et même radier.

Comme cela a été indiqué précédemment, plusieurs réacteurs de recherche français (MASURCA, RHF, RJH...) sont équipés d'accéléromètres qui émettent un signal vers le système de protection du réacteur. En cas de détection de sollicitations sismiques atteignant un certain niveau, le système de protection déclencherait alors l'arrêt d'urgence du réacteur, suffisamment tôt pour que les absorbants de sécurité soient suffisamment insérées dans la zone du cœur lorsque surviendraient les secousses les plus importantes.

Pour ce qui concerne les agressions d'origine climatique, les réacteurs de recherche sont des installations assez peu sensibles aux fortes chaleurs et aux grands froids. En

221. L'adoption de ce système d'isolation parasismique augmente l'amplitude des accélérations à la fréquence propre du système (environ $0,5 \mathrm{~Hz}$ ). La France dispose d'une expérience en la matière, les quatre tranches de la centrale de Cruas-Meysse ayant été installées (en 1977) sur un tel système d'isolation, le spectre sismique de sol excédant pour ce site, au-dessus de $2 \mathrm{~Hz}$, le spectre forfaitaire retenu par EDF pour les réacteurs du parc électronucléaire. Sur ces systèmes d'isolation sismique, voir la publication de l'AFCEN French Experience and Practice of Seismically Isolated Nuclear Facilities, PTAN RCC-CW 2015. 
particulier, l'évacuation de la puissance résiduelle d'un réacteur de recherche ne nécessite pas, pour beaucoup d'entre eux, de source froide extérieure (autre que l'air) et les besoins en électricité peuvent être, si besoin, réduits à la surveillance des principaux paramètres importants pour la sûreté.

Les bâtiments des réacteurs de recherche sont munis de paratonnerres et des parafoudres sont disposés au niveau des installations électriques.

\subsection{Dispositifs expérimentaux et équipements spécifiques aux réacteurs de recherche}

Les réacteurs de recherche ont la particularité d'accueillir des équipements ou dispositifs expérimentaux, dont certains peuvent être placés directement dans le cœur du réacteur ou à sa périphérie. Parmi ces équipements et dispositifs, on peut distinguer des dispositifs d'irradiation simples, des boucles expérimentales plus complexes, des canaux neutroniques, des « sources froides » et des « sources chaudes ». Comme cela a déjà été indiqué au paragraphe 7.1.2, les interactions de ces équipements et dispositifs avec le cœur du réacteur doivent être analysés du point de vue de la sûreté, bien évidemment dans les conditions normales de fonctionnement du réacteur, mais aussi dans les conditions incidentelles ou accidentelles susceptibles d'affecter aussi bien le réacteur que les dispositifs expérimentaux.

Pour un équipement ou dispositif expérimental, une telle analyse nécessite de bien identifier les différentes " sources de dangers » potentiels associées, susceptibles, dans des conditions dégradées pouvant aller par exemple jusques et y compris la rupture complète de cet équipement ou dispositif ou encore son " effacement " de la zone du cœur, d'avoir un effet négatif sur la sûreté du réacteur ou en termes de radioprotection. Ces sources de dangers sont la matière fissile, les matériaux absorbants, les produits radioactifs ou toxiques, les produits susceptibles d'exploser au contact de l'air, les produits susceptibles d'entraîner des réactions chimiques violentes au contact de l'eau, les fluides ou gaz sous pression, des matériaux à température élevée pouvant interagir avec l'eau du cœur du réacteur par interaction thermodynamique...

Les équipements et dispositifs expérimentaux sont en particulier susceptibles de modifier la réactivité du cœur en fonction des propriétés des matériaux qui les composent, ceux-ci pouvant avoir des propriétés d'absorption (matériaux neutrophages), de réflexion ou de modération neutroniques. Dès lors, en cas de mouvement intempestif (retrait incontrôlé du cœur par exemple), l'insertion de réactivité pouvant être occasionnée dans le cœur doit être maîtrisable par le système de protection du réacteur. Pour les dispositifs d'irradiation simple, cela peut être obtenu en limitant, par conception, le « poids » en réactivité de l'équipement ou du dispositif concerné (cette limite étant alors inscrite dans les spécifications techniques d'exploitation). Pour les dispositifs plus importants, dont le « poids " en réactivité ne peut pas être suffisamment limité, des dispositions constructives permettent de prévenir ou de limiter leur retrait incontrôlé (dispositifs d'accrochage, dispositifs anti-envol).

Pour les boucles expérimentales, d'autres risques peuvent être à considérer, en raison par exemple de la mise en œuvre de fluides sous pression (dans des conditions 
représentatives des réacteurs à eau sous pression par exemple - 155 bars [figure 7.12]) ou d'éléments réactifs tel que le sodium qui réagit violement au contact de l'eau. Ces boucles peuvent aussi être amenées à devoir contenir des matériaux en fusion lorsque cette fusion fait partie des objectifs poursuivis dans une expérience réalisée sur un élément combustible.

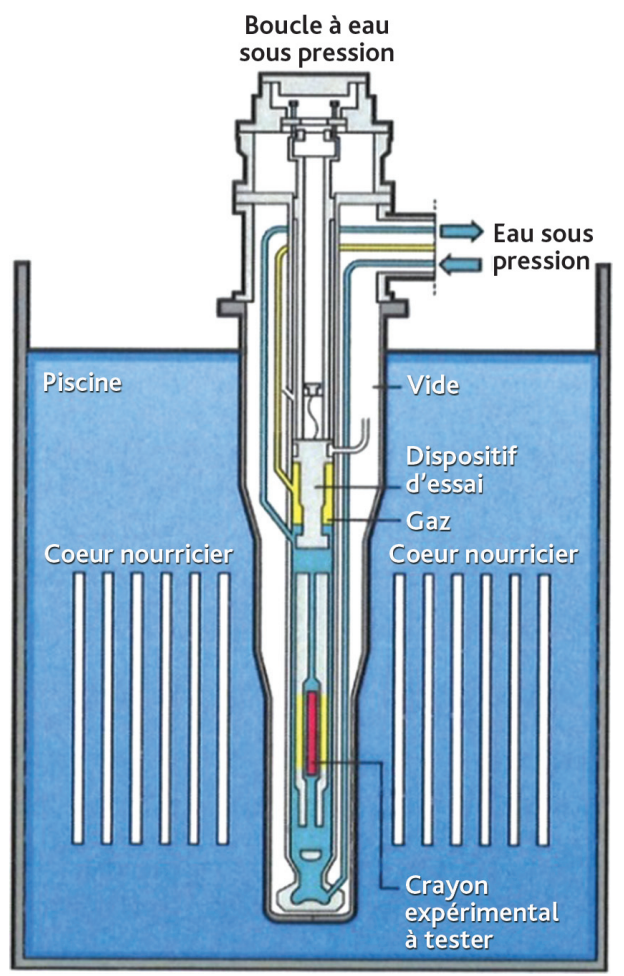

Figure 7.12. Schéma d'implantation de la boucle à eau sous pression dans le cœur du réacteur CABRI. (c) Stéphane Jungers/IRSN.

Des essais à caractère démonstratif sur maquettes peuvent apparaître nécessaires pour apprécier les effets de l'explosion d'une boucle expérimentale sur les éléments combustibles du cœur du réacteur au sein ou à proximité duquel elle est placée. On peut citer à ce sujet le cas de la boucle OTHELLO du réacteur OSIRIS, conçue pour la réalisation d'études relatives aux réacteurs à haute température (HTR), pour laquelle, dans la maquette, la boucle a été pré-entaillée pour rompre à la pression souhaitée.

Enfin, les « sources froides » et les « sources chaudes », utilisées dans les réacteurs à " faisceaux sortis de neutrons » contiennent généralement pour les premières de l'hydrogène $\left(\mathrm{H}_{2}\right)$ ou du deutérium $\left(\mathrm{D}_{2}\right)$ liquide et pour les secondes du graphite à plus de $1000^{\circ} \mathrm{C}$. Une défaillance de l'enveloppe (ou des enveloppes) de ces équipements conduirait à la mise en contact de leur contenu avec l'eau du cœur du réacteur, induisant en particulier un risque d'explosion d'hydrogène ou de deutérium, ou encore d'explosion de vapeur $^{222}$ pouvant affecter le cœur ou des « barrières » de confinement du réacteur.

222. Par interaction thermodynamique entre le graphite à $1000{ }^{\circ} \mathrm{C}$ et l'eau. 
La prévention de ce risque repose principalement, d'une part sur l'interposition de " barrières » ou enveloppes adaptées (en nombre et en capacité de résistance) entre la source de danger et le cœur du réacteur, d'autre part sur la maîtrise des paramètres propres à ces équipements spécifiques (pression de deutérium, températures...).

Enfin, un autre point important qui doit être examiné dès la conception des équipements ou dispositifs expérimentaux est le vieillissement des matériaux. Les flux de neutrons, en particulier, auxquels les matériaux qui les composent sont soumis modifient progressivement leurs propriétés mécaniques. Cela est particulièrement le cas, pour les réacteurs à " faisceaux sortis de neutrons ", des extrémités des canaux neutroniques (doigts de gant et tout particulièrement leur nez) qui sont en permanence au plus près du cœur du réacteur. Ces canaux sont généralement réalisés en aluminium ou alliage d'aluminium, ou encore composés de zirconium (comme le Zircaloy). Ces matériaux, utilisés pour leurs propriétés de « transparence » aux neutrons, se fragilisent sous irradiation neutronique. C'est pourquoi les équipements concernés (doigts de gant ou autres comme des caissons de cœurs) doivent être, le cas échéant, remplacés au cours de la vie d'un réacteur de recherche. La mise en œuvre de " plans de surveillance » d'échantillons ou d'éprouvettes réalisées dans les matériaux correspondants et irradiés dans des zones plus rapprochées du cœur (voire dans le cœur) que ne le sont les équipements peut permettre d'anticiper les effets du vieillissement et de définir les délais de remplacement de ces équipements.

\subsection{Radioprotection et effluents}

\subsubsection{Radioprotection}

Le système de radioprotection français repose sur trois grands principes, inscrits notamment dans le code de la santé publique :

- la justification des activités comportant un risque d'exposition à des rayonnements ionisants ;

- l'optimisation des expositions à ces rayonnements au niveau le plus faible raisonnablement possible en tenant compte des facteurs économiques et sociaux ;

- la limitation des doses d'expositions individuelles à ces rayonnements.

La réglementation nationale fixe en particulier des limites de doses individuelles annuelles pour les personnes du public et pour les travailleurs (tableau 7.4).

Le code du travail prévoit de plus que l'exploitant délimite, autour des sources de rayonnements ionisants, des zones surveillées et réglementées. Ces zones sont définies dans l'arrêté « zonage » du 15 mai 2006 (tableau 7.5).

Dans un réacteur de recherche (et ses installations associées), les sources de rayonnements ionisants sont multiples : éléments combustibles, faisceaux de neutrons, sources neutroniques de démarrage, sources d'étalonnage, sources utilisées pour des contrôles radiographiques... Les activités d'exploitation pouvant conduire à l'exposition de personnes sont par ailleurs variées :

- chargement ou déchargement du cœur du réacteur, 
Tableau 7.4. Limites d'exposition pour les personnes du public et pour les travailleurs, du fait des activités nucléaires, indiquées dans le code de la santé publique (article R. 1333-8) et dans le code du travail (article R. 4451-13).

\begin{tabular}{|l|c|c|}
\hline Type de dose & $\begin{array}{c}\text { Personnes } \\
\text { du public } \\
\text { par an }\end{array}$ & $\begin{array}{c}\text { Travailleurs exposés } \\
\text { (cas des adultes) } \\
\text { sur douze mois consécutifs }\end{array}$ \\
\hline Dose efficace annuelle & $1 \mathrm{mSv}$ & $20 \mathrm{mSv}$ \\
\hline $\begin{array}{l}\text { Dose équivalente à la peau, aux avant-bras, aux } \\
\text { pieds et aux chevilles (dose moyenne pour une } \\
\text { surface de } 1 \mathrm{~cm}^{2} \text { ) }\end{array}$ & $50 \mathrm{mSv}$ & $500 \mathrm{mSv}$ \\
\hline Dose équivalente au cristallin & $15 \mathrm{mSv}$ & $150 \mathrm{mSv}^{223}$ \\
\hline
\end{tabular}

Tableau 7.5. Limites des zones surveillées et règlementées définies dans l'arrêté « zonage » (hors irradiation naturelle).

\begin{tabular}{|l|c|c|}
\hline Type de zone & Couleur & Dose efficace \\
\hline Zone non réglementée & & $<0,080 \mathrm{mSv} / \mathrm{mois}$ \\
\hline Zone surveillée & & $<0,0075 \mathrm{mSv} / \mathrm{h}$ \\
\hline Zone contrôlée & & $<0,025 \mathrm{mSv} / \mathrm{h}$ \\
\hline $\begin{array}{l}\text { Zone contrôlée spécialement } \\
\text { réglementée }\end{array}$ & & $<2 \mathrm{mSv} / \mathrm{h}$ \\
\hline & & $<100 \mathrm{mSv} / \mathrm{h}$ \\
\hline Zone contrôlée interdite & $\geq 100 \mathrm{mSv} / \mathrm{h}$ \\
\hline
\end{tabular}

- chargement, déchargement ou modification de dispositifs d'irradiation ou de boucles expérimentales,

- préparation d'assemblages ou d'éléments combustibles, d'expériences d'irradiation ou d'activation,

- réalisation de mesures sur des matériaux ou des combustibles irradiés,

- contrôles en service...

Sur la base de l'analyse des activités et des caractéristiques des sources de rayonnements ionisants, l'exploitant classe les locaux de son installation en zones « non réglementées 》, « réglementées 》, « spécialement réglementées 》 ou encore « interdites » où devront être respectées les limites indiquées plus haut, grâce à la mise

223. Les valeurs indiquées ici correspondent à celles de la publication $n^{\circ} 60$ de la CIPR parue en 1991 . Les valeurs recommandées dans la publication n 103 de la CIPR, fin 2007, sont de 15 mSv pour les personnes du public et $20 \mathrm{mSv}$ pour les travailleurs. Elles ont été intégrées en 2018 dans la réglementation française (code de l'environnement, code de la santé, code du travail). 


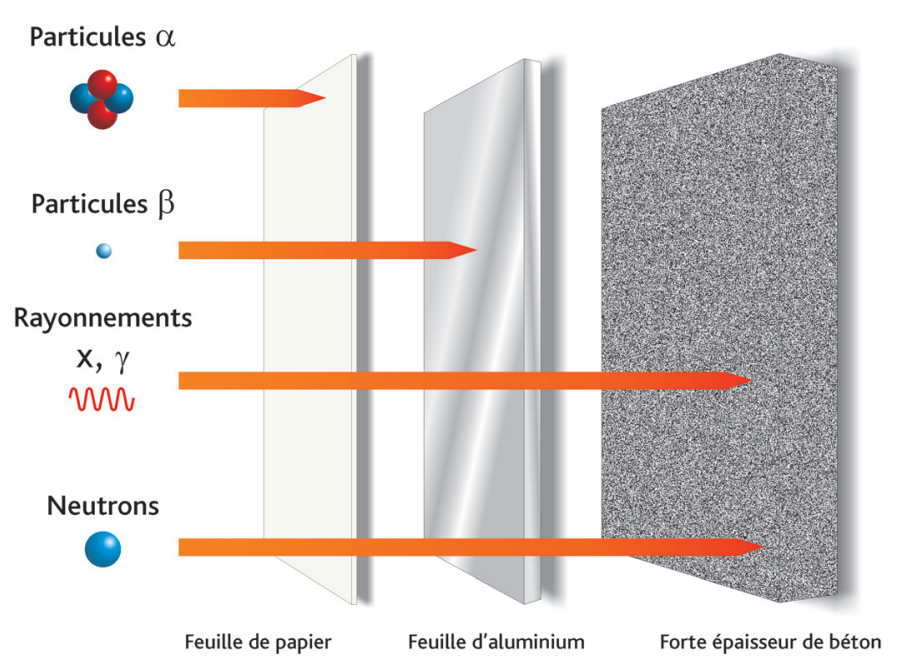

Figure 7.13. Efficacité de quelques protections à l'égard des différents types de rayonnements ionisants. (c) Georges Goué/IRSN.

en œuvre de dispositions techniques - quelques types de protection physique sont illustrés sur la figure 7.13 - et organisationnelles. Des blocs de béton ou des parois en plomb sont souvent utilisés à cette fin (par exemple autour des guides de neutrons dans les espaces dédiés aux physiciens menant des expériences). Pour des opérations portant sur des petits objets irradiés, il s'agira de briques de béton ou de plomb placés autour des objets auxquels on veut avoir accès tout en se protégeant des rayonnements ionisants. Dans certains cas, les opérateurs peuvent être amenés à porter des tabliers de plomb.

Pour vérifier que les limites de doses qui leur sont applicables sont bien respectées, les travailleurs portent en permanence un dispositif de mesure adapté à la nature des rayonnements ionisants, qui permet d'assurer un suivi dosimétrique. Une dosimétrie opérationnelle ${ }^{224}$ permet également de les alerter en cas de dépassement d'un débit de dose prédéterminé ou d'une dose pour une certaine durée.

Lorsque des travaux sont nécessaires sur l'installation, une analyse de sûreté est menée et comporte un volet radioprotection. En particulier, une étude d'optimisation en radioprotection est réalisée, visant à maintenir les doses individuelles, le nombre de personnes exposées et la probabilité d'occurrence d'expositions fortuites pendant l'intervention aussi faibles que raisonnablement possible, compte tenu des facteurs économiques et sociétaux. Les principales étapes sont les suivantes :

224. La dosimétrie opérationnelle, aussi appelée dosimétrie active, consiste en une mesure en temps réel de l'exposition externe à l'aide d'un dosimètre individuel opérationnel. Elle est mise en œuvre par la personne compétente en radioprotection (PCR) sous la responsabilité du chef d'établissement. Le système électronique d'un dosimètre opérationnel permet une lecture immédiate de la dose reçue par le travailleur. Ce dosimètre permet une mesure lors d'une tâche spécifique ou sur une période donnée (entrée - sortie en zone contrôlée). Munis d'alarmes auditives ou visuelles, il se déclenche en cas de dépassement de doses prédéfinies selon les besoins, ce qui permet au porteur de connaître en permanence le risque radioactif auquel il est soumis. Le porteur ou, grâce à la télétransmission, le service compétent peut suivre et optimiser l'exposition durant l'exposition même. 
- évaluation de la situation d'exposition, y compris les expositions potentielles (celles qui pourraient survenir si l'opération ne se passait pas comme prévu) ;

- sélection d'une borne supérieure appropriée pour restreindre les doses ;

- identification des options de protection possibles ;

- sélection de la meilleure option dans les circonstances en présence ;

- mise en œuvre de l'option choisie.

Le retour d'expérience est pris en compte. Les résultats sont évalués en particulier dans la perspective de futures opérations de même type.

L'optimisation de la protection radiologique n'est pas impérativement une minimisation des doses. La protection optimisée est le résultat d'une évaluation et d'un dialogue, qui comparent soigneusement les risques liés à l'exposition envisagée et les ressources disponibles pour la protection des individus. Ainsi la meilleure option n'est pas nécessairement celle correspondant aux doses les plus faibles. De surcroît, la protection radiologique ne se limite pas aux expositions individuelles, le nombre d'individus exposés doit également être pris en compte. La dose efficace collective est un paramètre-clé de l'optimisation de la protection des travailleurs. La comparaison des options de protection dans un objectif d'optimisation doit entraîner la considération attentive des caractéristiques de la distribution des expositions individuelles au sein de la population exposée.

Les équipements ou dispositifs expérimentaux peuvent également être des sources de rayonnements ionisants. C'est le cas des boucles expérimentales dans lesquelles des combustibles sont soumis à des transitoires pouvant conduire à des ruptures de gaines voire à la fusion de combustible et contaminer la boucle d'essais concernée. Les composants de ces boucles sont équipés de protections biologiques et des équipements spécifiques de manutention permettent de limiter les rayonnements ionisants dans l'installation (hotte de transfert...).

\subsubsection{Effluents}

Globalement, la gestion des effluents résultant de l'exploitation d'un réacteur de recherche est semblable à celle des effluents de toute installation nucléaire de base. Il existe toutefois deux types d'effluents particuliers à mentionner ici :

- l'eau tritiée produite par capture de neutrons par le deutérium de l'eau lourde utilisée dans les réacteurs à « faisceaux sortis de neutrons » (RHF et ORPHÉE) ;

- les effluents provenant des boucles expérimentales dans lesquelles une fusion de combustible expérimental peut se produire, voire être recherchée.

La gestion de ces effluents particuliers fait l'objet de dispositions spécifiques de traitement (installation de détritiation par exemple pour ORPHEE, installation de dépotage de fûts d'eau lourde pour le RHF en vue d'un traitement de l'eau lourde dans une autre installation).

Des ordres de grandeur de rejets d'effluents de réacteurs de recherche français sont indiqués dans le tableau 7.6 , avec les limites annuelles fixées par arrêtés ou décisions. 
Tableau 7.6. Rejets de deux réacteurs de recherche (RHF, OSIRIS-ISIS) et limites annuelles.

\begin{tabular}{|c|c|c|}
\hline \multicolumn{3}{|c|}{ Réacteur à haut flux de Grenoble (RHF) } \\
\hline Type de rejets & $\begin{array}{l}\text { Valeur maximale annuelle sur la } \\
\text { période } 2010-2015 \\
\text { Effluents gazeux (GBq) } \\
\text { Effluents liquides (GBq) }\end{array}$ & $\begin{array}{l}\text { Limites annuelles } \\
\text { (arrêté du 3/08/2007) }\end{array}$ \\
\hline Gaz rares & 1200 & 10000 \\
\hline Tritium & $\begin{array}{c}12000 \\
370\end{array}$ & $\begin{array}{c}75000 \\
1000\end{array}$ \\
\hline Carbone 14 & $\begin{array}{c}460 \\
0,3\end{array}$ & $\begin{array}{c}2000 \\
1,5\end{array}$ \\
\hline lodes & $\begin{array}{l}3,4 \cdot 10^{-3} \\
1,3 \cdot 10^{-3}\end{array}$ & $\begin{array}{c}1 \\
0,1\end{array}$ \\
\hline Autres émetteurs $\beta \gamma$, aérosols & $\begin{array}{c}3,1.10^{-4} \\
0,13\end{array}$ & $\begin{array}{c}0,1 \\
1\end{array}$ \\
\hline \multicolumn{3}{|c|}{ Réacteurs OSIRIS-ISIS (INB n40) } \\
\hline Type de rejets & $\begin{array}{l}\text { Valeur maximale annuelle sur la } \\
\text { période } 2010-2015 \\
\text { Effluents gazeux (GBq) } \\
\text { Effluents liquides }(\mathrm{GBq})\left(^{*}\right)\end{array}$ & $\begin{array}{c}\text { Limites annuelles } \\
\text { (décision n²009-DC- } \\
0156 \text { de l'ASN, } \\
\text { du 15/09/2009) }\end{array}$ \\
\hline Gaz rares & $\begin{array}{c}7356 \\
-\end{array}$ & $\begin{array}{c}10000 \\
-\end{array}$ \\
\hline Tritium & $\begin{array}{c}297 \\
3,4.10^{-3}\end{array}$ & $\begin{array}{c}2000 \\
0,5\end{array}$ \\
\hline Carbone 14 & $\begin{array}{c}1,14 \\
7,6.10^{-4}\end{array}$ & $\begin{array}{c}20 \\
10^{-2}\end{array}$ \\
\hline lodes & $4,5 \cdot 10^{-4}$ & 0,5 \\
\hline Autres émetteurs $\beta \gamma$ & $\begin{array}{l}6,3 \cdot 10^{-4} \\
2,3 \cdot 10^{-3}\end{array}$ & $\begin{array}{c}0,01 \\
2.10^{-2}\end{array}$ \\
\hline Émetteurs $\alpha$ & $\stackrel{-}{1,7.10^{-4}}$ & $5.10^{-3}$ \\
\hline
\end{tabular}

${ }^{(*)}$ Effluents liquides rejetés dans le réseau des effluents chimiques. Des effluents actifs liquides sont aussi envoyés aux stations de traitement de Saclay et de Marcoule.

\subsection{Dispositions de préparation aux situations d'urgence et de gestion de telles situations (gestion de crise)}

L'hypothèse de rejets (significatifs) de substances radioactives qui conduiraient à la mise en œuvre de mesures de protection des populations (évacuation, confinement 
dans les maisons fermées, distribution de tablettes d'iode stable, restrictions de consommation de produits alimentaires...) suppose l'échec ou une efficacité insuffisante des quatre premiers niveaux de la défense en profondeur. Elle conduit à la définition de dispositions spécifiques dans le cadre de l'organisation générale de crise au niveau national, qui s'applique à toutes les installations nucléaires de base, dont les réacteurs de recherche. Cette organisation ne sera pas développée dans le cadre du présent ouvrage ${ }^{225}$, seuls les aspects intéressants plus particulièrement les réacteurs de recherche seront mentionnés.

Parmi les dispositions de crise, figurent les PUI, plans d'urgence interne dont l'activation $^{226}$ est de la responsabilité des exploitants, et les PPI, plans particuliers d'intervention du ressort des pouvoirs publics - ces plans d'urgence ont globalement vu le jour au début des années 1980, leur nécessité ayant été confortée à la lumière des enseignements tirés de l'accident survenu en 1979 à la centrale nucléaire de Three Mile Island aux États-Unis ${ }^{227}$. Pour les réacteurs de recherche exploités par le CEA, c'est le Directeur du centre concerné ou son représentant (ou encore le cadre d'astreinte de Direction en dehors des heures ouvrables) qui déclenche le PUI. Pour le RHF, c'est le Chef de la division réacteur de l'Institut Laue-Langevin ou son adjoint (ou encore l'ingénieur de service d'astreinte) qui déclenche le PUI. Dans tous les cas, l'alerte des autorités doit être effectuée dans un délai inférieur à deux heures.

Le PUI définit, sur la base d'une étude figurant dans le rapport de sûreté (voir ciaprès), les mesures d'organisation, les méthodes d'intervention et les moyens nécessaires que l'exploitant met en œuvre en cas de situation d'urgence pour protéger des rayonnements ionisants le personnel, le public et l'environnement et préserver ou rétablir la sûreté de l'installation. Il peut aussi préciser les modalités de mise en œuvre de mesures incombant à l'exploitant en application du PPI (alerte et mise à l'abri en « mode réflexe $»)$.

Dans le cadre de la mise en œuvre d'un PUI par un exploitant, celui-ci dresse régulièrement un état de la situation de son installation accidentée, et effectue un pronostic permettant d'anticiper l'évolution possible de cette situation. Ces éléments sont partagés et discutés avec l'Autorité de sûreté nucléaire et l'IRSN. L'un des « outils de crise » est l'approche diagnostic-pronostic dite 3D-3P 228 mise au point dans les années 1990 pour les centrales du parc électronucléaire français (par l'IPSN et EDF). Il est dans le principe applicable aux réacteurs de recherche français, avec toutefois quelques adaptations nécessaires pour tenir compte de leurs particularités (notamment sur l'agencement et le nombre de leurs «barrières » de confinement).

D’autres « outils de crise » existent (autres que des logiciels simplifiés de simulation voir le chapitre 11), tels que des documents synthétiques relatifs à des « accidents-types ».

225. Voir par exemple : « La gestion d'une crise nucléaire : des responsabilités partagées » sur le site internet de l'ASN, ou encore " Face à un accident nucléaire ", IRSN, Collection Livrets des professionnels - Décembre 2008.

226. Selon le concept de défense en profondeur, le PUI relève du $4^{\mathrm{e}}$ niveau, supposant l'échec des trois premiers.

227. Ouvrage cité au nota 152.

228. Le chiffre 3 se réfère aux trois « barrières » de confinement des réacteurs à eau sous pression. 
En effet, l'exploitant d'un réacteur de recherche est amené à considérer, en fonction des caractéristiques de son installation et des risques associés à son environnement, un ou plusieurs accidents représentatifs - aussi appelés accidents-types - pour lesquels des dispositions de gestion de crise seraient à mettre en place. Ces accidents-types sont choisis parmi les accidents étudiés dans le cadre notamment de la démonstration de sûreté et sont présentés dans un chapitre du rapport de sûreté ${ }^{229}$. Ces accidents-types représentent les différents potentiels de dangers de l'installation et ne sont pas exclusivement de nature radiologique (des accidents ayant des conséquences chimiques peuvent par exemple être retenus). Pour de nombreux réacteurs de recherche français, l'accident de type BORAX fait partie de ces accidents-types, car il conduit à une fusion dans le cœur et à une surpression dans le bâtiment du réacteur, pouvant entraînant des rejets dans l'environnement. Mais des accidents moins sévères peuvent aussi être retenus dans les $\mathrm{PUI}^{230}$. Parmi les autres accidents-types, on peut citer :

- la rupture de gaine d'une plaque de combustible sous eau, dans le cœur du réacteur ;

- la fusion d'une plaque de combustible sous eau, dans le cœur du réacteur ;

- la fusion à l'air d'un élément combustible dans le bâtiment du réacteur, lors d'un déchargement du cœur du réacteur ;

- la chute d'un emballage de transport contenant plusieurs éléments combustibles...

Certains de ces accidents-types peuvent supposer de multiples défaillances internes ou la survenue d'une agression naturelle d'une intensité supérieure à celle considérée à la conception de l'installation.

En cas d'accident impliquant une dégradation importante de combustible, le personnel d'exploitation et les chercheurs présents dans le bâtiment du réacteur et les locaux attenants, tels que la salle de commande, peuvent devoir être évacués compte tenu des débits de dose atteints. Il est également à noter que, dans le cas d'une fusion du cœur à l'air (en cas de découvrement de celui-ci) ou d'élément combustible en manutention dans le bâtiment d'un réacteur, l'irradiation directe à l'extérieur du bâtiment du réacteur pourra aussi être importante. C'est pourquoi des réacteurs de recherche français sont dotés d'un poste de repli implanté à une distance suffisante du réacteur au regard des débits de dose pouvant résulter de l'installation accidentée. Ce poste de repli comprend les informations nécessaires à la gestion de l'accident (paramètres neutroniques et thermohydrauliques du cœur du réacteur, niveaux d'eau, débits de dose, mesure en continu de l'activité rejetée par la cheminée...). De même, certains systèmes de l'installation peuvent être commandés à partir du poste de repli, tels que la ventilation de sauvegarde permettant de « piloter » les rejets dans l'atmosphère en situation accidentelle.

229. Prescrit dans le décret $n^{\circ}$ 2007-1557 du 2 novembre 2007, dit décret " procédures ».

230. Ainsi, par rapport à l'INSAG-10, un exploitant (en France) peut être amené à prendre la décision de déclencher son PUI pour des accidents envisagés au titre du niveau 3 de la défense en profondeur (l'accident de type BORAX se situant au niveau 4). 
Les accidents-types font l'objet (notamment à l'IRSN) de documents synthétiques tenus à jour où sont précisées les conséquences possibles de chacun d'entre eux, avec différentes variantes en termes de conditions météorologiques, de temps écoulé entre l'arrêt du réacteur et l'accident ${ }^{231}$, de configuration des systèmes de ventilation, d'aggravants possibles considérés dans le cadre du volet " pronostic » de la méthode 3D-3P (par exemple pièges à iode en service ou hors service)...

Il est à noter que des fiches synthétiques descriptives des réacteurs de recherche sont également à disposition des équipes de crise, précisant les inventaires radiologiques dans les cœurs et les piscines d'entreposage de combustible usé, les caractéristiques des « barrières » de confinement et des systèmes comme ceux de ventilation et de filtration... Ces fiches sont indispensables pour les réacteurs de recherche du fait du nombre relativement restreint de personnes ayant une connaissance approfondie de ces réacteurs.

Enfin, concernant les PPI, les conditions d'une évacuation ou d'un confinement de populations sont étudiées par les pouvoirs publics. Elles sont complétées par la préparation de mesures de contrôle de consommation ou de commercialisation à court, moyen ou long termes de produits alimentaires éventuellement contaminés. La mise en œuvre de ces mesures est du ressort du Préfet du département concerné.

\subsection{Aspects de sûreté concernant le démantèlement des réacteurs de recherche}

Selon les termes de la règlementation applicable, l'exploitant d'un réacteur de recherche doit désormais présenter, dès la demande d'autorisation de création d'une telle installation, les principes généraux et les dispositions relatifs au démantèlement futur de l'installation (" plan de démantèlement »). Ces éléments doivent être, le cas échéant, actualisés lors de la demande d'autorisation de mise en service de l'installation, ainsi que lors des réexamens de sûreté. Enfin, comme pour toute installation nucléaire de base, l'exploitant qui envisage la mise à l'arrêt définitif et le démantèlement effectif d'un réacteur de recherche doit déposer en temps voulu un dossier spécifique.

En France, plusieurs réacteurs de recherche ont déjà été démantelés, comme le réacteur universitaire de Strasbourg (RUS) ou HARMONIE ; le réacteur SILOE à Grenoble est désormais déclassé et le réacteur d'enseignement ULYSSE à Saclay est en phase avancée de démantèlement. Un certain nombre d'autres réacteurs sont en cours de déconstruction, sachant que la stratégie retenue vise un démantèlement « immédiat ${ }^{232}$ »

231. Cela détermine la puissance résiduelle à prendre en compte, qui décroît de façon globalement exponentielle dans le temps.

232. Le guide $n^{\circ} 6$ de l'ASN (version révisée du 30 août 2016), relatif à l'arrêt définitif, au démantèlement et au déclassement des INB, rappelle à cet égard le code de l'environnement, qui prescrit que l'exploitant d'une installation nucléaire de base « procède à son démantèlement dans un délai aussi court que possible, dans des conditions économiquement acceptables et dans le respect des principes énoncés à l'article L. 1333-1 du code de la santé publique et au II de l'article L. 110-1 du présent code ". 
après l'arrêt définitif du réacteur - de façon, notamment, à pouvoir utiliser, autant que possible, les compétences et les connaissances des opérateurs encore présents. Ainsi, le démantèlement du réacteur PHENIX a été prescrit dans la continuité de la décision de sa mise à l'arrêt par le CEA.

La réglementation actuelle met l'accent sur la prise en compte du démantèlement au plus tôt de la vie d'une installation, dès la conception, en vue de faciliter les opérations de déconstruction et de minimiser les risques associés. Sur ce point, on peut noter que pour les réacteurs à « faisceaux sortis de neutrons » (ORPHEE, RHF), le démontage complet de la cuve du bloc-pile avait été prévu dès la conception de ces réacteurs. 